\title{
$\alpha$-Amidation of Cyclic Ethers Catalyzed by Simple Copper Salt and A Mild and Efficient Preparation Method for $\alpha, \varpi$-Aminoalcohols
}

\author{
Ling $\mathrm{He}^{\mathrm{a}, \mathrm{b}}$, Jing $\mathrm{Yu}^{\mathrm{b}}$, Ji Zhang ${ }^{\mathrm{a}}$, Xiao-Qi $\mathrm{Yu}^{\mathrm{a}_{*}}$ \\ a. Department of Chemistry, Key Laboratory of Green Chemistry and Technology (Ministry of Education), \\ Sichuan University, 29 Wangjiang Road, Chengdu, Sichuan 610064, P. R. China \\ b. Key Laboratory of Drug-Targeting of Education Ministry of China, West China School of Pharmacy, Sichuan \\ University,No.17, Section3,South Renmin Road, Chengdu, Sichuan 610041, P. R. China;
}

\section{Supporting Information}

\section{General Experimental Section:}

All catalyses were performed under nitrogen and argon atmosphere. Silica gel F254 plates were used for TLC and spots were examined under UV light at $254 \mathrm{~nm}$ and developed by an iodine vapor. Flash chromatography was performed on silica gel H. Solvents were purified according to standard procedures. ${ }^{1} \mathrm{H}$ and ${ }^{13} \mathrm{C}$ NMR spectra were recorded on Bruker AC-E $200 \mathrm{MHz}$, Varian Mercury 400 $\mathrm{MHz}$ and Bruker Avance $600 \mathrm{MHz}$ spectrometer, using $\mathrm{CDCl}_{3}$ as the solvent. The mass spectra (ESI / HRMS) were recorded on a Bruker Daltonics Data analysis 3.2 mass spectrometer.

\section{General Procedure for the Amidation of Cyclic Ethers:}

A reaction flask was charged with substrate, iodosobenzene diacetate, p-tolulensulfonamide, catalyst $(10 \% \mathrm{~mol})$ and dichloromethane, and then the mixture was stirred at $40{ }^{\circ} \mathrm{C}$ under nitrogen atmosphere for about $4 \mathrm{~h}$. After cooling to room temperature, the resulting mixture was filtered; the filtrate was concentrated in vacuo. The residue was purified by column chromatography on silica gel with petroleum ether/ethyl acetate or dichloromethane/acetone as eluent to afford product of amidation.

\section{General Procedure for Cleavage of products of amidation:}

A mixture of tosylamide 2, sodium borohydride and tetrahydrofuran was stirred at room temperature under nitrogen atmosphere for about $2 \sim 4 \mathrm{~h}$. To the resulting mixture, $20 \mathrm{ml}$ of saturated $\mathrm{NH}_{4} \mathrm{Cl}$ were added and extracted with ethyl acetate $(20 \mathrm{ml} \times 3)$, the organic layer was dried with $\mathrm{Na}_{2} \mathrm{SO}_{4}$ and concentrated in vacuo. The residue was purified by column chromatography on silica gel with petroleum ether/ethyl acetate or dichloromethane/acetone as eluent to give $\omega$-amino-alcohols. 


\section{Characterization of compounds:}<smiles>[SH2-]NC1CCCO1</smiles>

Tetrahydrofuran-2-yl-tolylsulfonamide

${ }^{1} \mathrm{H}-\mathrm{NMR}\left(\mathrm{CDCl}_{3}, 300 \mathrm{MHz}\right) \delta(\mathrm{ppm})$

${ }^{13} \mathrm{C}-\mathrm{NMR}\left(\mathrm{CDCl}_{3}, 75 \mathrm{MHz}\right) \delta(\mathrm{ppm})$

MS (ESI-) $\mathrm{m} / \mathrm{z}(\%)$ for $\mathrm{C}_{11} \mathrm{H}_{13} \mathrm{NO}_{3} \mathrm{~S}(\mathrm{M}-\mathrm{H})$<smiles>[SbH3]C1CCCCO1</smiles>

Cycloxypentane-2-yl-tolylsulfonamide

${ }^{1} \mathrm{H}-\mathrm{NMR}\left(\mathrm{CDCl}_{3}, 400 \mathrm{MHz}\right) \delta(\mathrm{ppm})$

${ }^{13} \mathrm{C}-\mathrm{NMR}\left(\mathrm{CDCl}_{3}, 75 \mathrm{MHz}\right) \delta(\mathrm{ppm})$

HRMS (ESI+) m/z (\%) for $\mathrm{C}_{12} \mathrm{H}_{17} \mathrm{NO}_{3} \mathrm{~S} \mathrm{Na}(\mathrm{M}+\mathrm{Na})$
$1.73 \sim 1.84(\mathrm{~m}, 3 \mathrm{H}), 1.86 \sim 1.89(\mathrm{~m}, 1 \mathrm{H}), 2.44(\mathrm{~s}$, $3 \mathrm{H}), 3.68 \sim 3.73(\mathrm{~m}, 2 \mathrm{H}), 5.29 \sim 5.39(\mathrm{~m}, 1 \mathrm{H})$ $7.28(\mathrm{~d}, J=8.0 \mathrm{~Hz}, 2 \mathrm{H}), 7.79(\mathrm{~d}, J=8.0$ $\mathrm{Hz}, 2 \mathrm{H})$.

21.5, 23.9, 32.5, 67.1, 84.9, 126.9, 129.5, 138.5, 143.3 .

Calcd. 240.0. Found 240.0.
$1.33 \sim 1.38 \quad(\mathrm{~m}, \quad 2 \mathrm{H}), \quad 1.45 \sim 1.54 \quad(\mathrm{~m}, 1 \mathrm{H})$, $1.78 \sim 1.84(\mathrm{~m}, 2 \mathrm{H}), 2.40(\mathrm{~s}, 3 \mathrm{H}), 3.38 \sim 3.40(\mathrm{~m}$, $1 \mathrm{H}), 3.68(\mathrm{~d}, 1 \mathrm{H}), 4.76\left(\mathrm{td}, J_{1}=2.0, J_{2}=9.2,1 \mathrm{H}\right)$, $5.40(\mathrm{~d}, J=9.2 \mathrm{~Hz}, 1 \mathrm{H}), 7.26(\mathrm{~d}, J=8.0 \mathrm{~Hz}, 2 \mathrm{H})$, $7.78(\mathrm{~d}, J=8.0 \mathrm{~Hz}, 2 \mathrm{H})$.

21.5, 22.3, 24.7, 31.8, 66.2, 82.0, 127.1, 129.4, 138.8, 143.2.

Calcd. 278.0821. Found 278.0826.<smiles>[NH3+]C1COCCO1</smiles>

\section{1,4-dioxyhexane-2-yl-tolylsulfonamide}

${ }^{1} \mathrm{H}-\mathrm{NMR}\left(\mathrm{CDCl}_{3}, 400 \mathrm{MHz}\right) \delta(\mathrm{ppm})$

2. $41(\mathrm{~s}, 3 \mathrm{H}), 3.40 \sim 3.44(\mathrm{~m}, 1 \mathrm{H}), 3.51 \sim 3.60(\mathrm{~m}$, $3 \mathrm{H}), 3.77 \sim 3.81(\mathrm{dd}, J=2.4 \mathrm{~Hz}$ and $12.6 \mathrm{~Hz}, 1 \mathrm{H})$, $4.96(\mathrm{~m}, 1 \mathrm{H}), 6.17(\mathrm{~d}, J=9.6 \mathrm{~Hz}, 1 \mathrm{H}), 7.28$ (d, $J$ $=8 \mathrm{~Hz}, 2 \mathrm{H}), 7.80(\mathrm{~d}, J=8 \mathrm{~Hz}, 2 \mathrm{H})$.

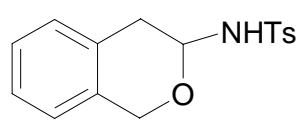

\section{Cycloxypentane-2-yl-tolylsulfonamide}


${ }^{1} \mathrm{H}-\mathrm{NMR}\left(\mathrm{CDCl}_{3}, 400 \mathrm{MHz}\right) \delta(\mathrm{ppm})$

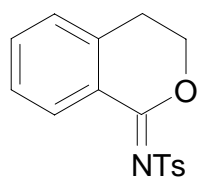

${ }^{1} \mathrm{H}-\mathrm{NMR}\left(\mathrm{CDCl}_{3}, 400 \mathrm{MHz}\right) \delta(\mathrm{ppm})$

${ }^{13} \mathrm{C}-\mathrm{NMR}-\mathrm{Dept}\left(\mathrm{CDCl}_{3}, 75 \mathrm{MHz}\right) \delta(\mathrm{ppm})$

${ }^{13} \mathrm{C}-\mathrm{NMR}\left(\mathrm{CDCl}_{3}, 75 \mathrm{MHz}\right) \delta(\mathrm{ppm})$<smiles>OCN[SbH3]</smiles>

${ }^{1} \mathrm{H}-\mathrm{NMR}\left(\mathrm{CDCl}_{3}, 400 \mathrm{MHz}\right) \delta(\mathrm{ppm})$

${ }^{13} \mathrm{C}-\mathrm{NMR}\left(\mathrm{CDCl}_{3}, 100 \mathrm{MHz}\right) \delta(\mathrm{ppm})$

MS (ESI+) m/z (\%) for $\mathrm{C}_{11} \mathrm{H}_{18} \mathrm{NO}_{3} \mathrm{~S}(\mathrm{M}+\mathrm{H})$<smiles>[NH-]CCCCO</smiles>

${ }^{1} \mathrm{H}-\mathrm{NMR}\left(\mathrm{CDCl}_{3}, 400 \mathrm{MHz}\right) \delta(\mathrm{ppm})$

${ }^{13} \mathrm{C}-\mathrm{NMR}\left(\mathrm{CDCl}_{3}, 100 \mathrm{MHz}\right) \delta(\mathrm{ppm})$ $2.43(\mathrm{~s}, 3 \mathrm{H}), 2.57 \sim 2.64(\mathrm{dt}, 1 \mathrm{H}), 2.81 \sim 2.89(\mathrm{~m}$, $1 \mathrm{H}), 3.59 \sim 3.71(\mathrm{~m}, 2 \mathrm{H}), 5.43(\mathrm{~d}, J=8.4 \mathrm{~Hz}, 1 \mathrm{H})$, $6.10(\mathrm{~d}, J=8.8 \mathrm{~Hz}, 1 \mathrm{H}), 7.11(\mathrm{~d}, J=5.2 \mathrm{~Hz}, 1 \mathrm{H})$, 7.19 7.22 (m.2H), 7.31 (d, J=8.4Hz, 2H), 7.86 (d, $J=8.4 \mathrm{~Hz}, 2 \mathrm{H})$

Calcd. 326.0821. Found 326.0824.

$2.42(\mathrm{~s}, 3 \mathrm{H}), 3.07(\mathrm{t}, 2 \mathrm{H}), 4.57(\mathrm{t}, 2 \mathrm{H})$, 7.22-7.35 (m, 2H), $7.31(\mathrm{~d}, J=8.4 \mathrm{~Hz}, 2 \mathrm{H}), 7.52$ (td, $J=1.2$ and $0.8 \mathrm{~Hz}, 1 \mathrm{H}), 7.92(\mathrm{~d}, J=8.4 \mathrm{~Hz}$, $2 \mathrm{H}), 8.08(\mathrm{~d}, J=8.0 \mathrm{~Hz}, 1 \mathrm{H})$.

21.6, $27.2(-), 68.8(-), 127.3,127.6,127.8$, $129.1,130.1,134.2$.

$21.5,27.2,68.6,125.2,127.3,127.6,127.8$, 129.1, 130.1, 134.1, 137.9, 139.2, 142.9, 162.8.

1.54 1.67 (m, 4H), 2. 43 (s, 3H), 2.97 (q, 2H), $3.63(\mathrm{t}, 2 \mathrm{H}), 4.86(\mathrm{t}, 1 \mathrm{H}), 7.31(\mathrm{~d}, J=8.4 \mathrm{~Hz}$, $2 \mathrm{H}), 7.74(\mathrm{~d}, J=8.4 \mathrm{~Hz}, 2 \mathrm{H})$, 21.5, 26.2, 29.5, 42.9, 62.2, 127.1, 129.7, 143.3, 146.5 .

Calcd. 244.33. Found 244.4 (100\%).

$1.32 \sim 1.40(\mathrm{~m}, 2 \mathrm{H}), 1.48 \sim 1.55(\mathrm{~m}, 4 \mathrm{H}), 1.77$ (br,2H),2.43 (s, 3H), 2.93 (q, 2H), 3.59 (t, 2H), $4.82(\mathrm{br}, 1 \mathrm{H}), 7.29(\mathrm{~d}, J=8.4 \mathrm{~Hz}, 2 \mathrm{H}), 7.74(\mathrm{~d}$, $J=8.4 \mathrm{~Hz}, 2 \mathrm{H})$.

21.5, 22.6, 29.1, 31.8, 42.9, 62.3, 127.0, 129.6, $136.9,143.3$. 
HRMS (ESI+) m/z (\%) for $\mathrm{C}_{12} \mathrm{H}_{19} \mathrm{NO}_{3} \mathrm{SNa}(\mathrm{M}+\mathrm{Na}) \quad$ Calcd. 280.0978. Found 280.0981.<smiles>OCCOCC[AsH3-]</smiles>

${ }^{1} \mathrm{H}-\mathrm{NMR}\left(\mathrm{CDCl}_{3}, 600 \mathrm{MHz}\right) \delta(\mathrm{ppm})$

$2.42(\mathrm{~s}, 3 \mathrm{H}), 3.13(\mathrm{t}, 2 \mathrm{H}), 3.49(\mathrm{~m}, 4 \mathrm{H}), 3.69(\mathrm{t}$, 2H), 5.52 (br, 1H), 7.29 (d, $J=8.1 \mathrm{~Hz}, 2 \mathrm{H})$, 7.76(d, $J=8.1 \mathrm{~Hz}, 2 \mathrm{H})$.

${ }^{13} \mathrm{C}-\mathrm{NMR}\left(\mathrm{CDCl}_{3}, 150 \mathrm{MHz}\right) \delta(\mathrm{ppm})$

$21.5,42.9,61.6,69.3,72.2,127.1,129.7,137.0$ 143.4 .

HRMS (ESI+) m/z (\%) for $\mathrm{C}_{11} \mathrm{H}_{17} \mathrm{NO}_{4} \mathrm{SNa}(\mathrm{M}+\mathrm{Na})$

Calcd. 282.0784. Found 282.0793.

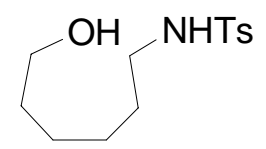

${ }^{1} \mathrm{H}-\mathrm{NMR}\left(\mathrm{CDCl}_{3}, 600 \mathrm{MHz}\right) \delta(\mathrm{ppm})$

$1.26 \sim 1.33(\mathrm{~m}, 4 \mathrm{H}), 1.43 \sim 1.54(\mathrm{~m}, 4 \mathrm{H}), 2.43(\mathrm{~s}, 3 \mathrm{H})$, $2.92(\mathrm{t}, 2 \mathrm{H}), 3.59$ (t, 2H),7. 29 (d, $J=8.4 \mathrm{~Hz}$, $2 \mathrm{H}), 7.75$ (d, $J=8.4 \mathrm{~Hz}, 2 \mathrm{H})$.

${ }^{13} \mathrm{C}-\mathrm{NMR}\left(\mathrm{CDCl}_{3}, 150 \mathrm{MHz}\right) \delta(\mathrm{ppm})$

21.5, 25.1, 26.1, 29.4, 32.3, 43.0, 62.6, 127.1, 129.7, 136.9, 143.3.

HRMS (ESI+) m/z (\%) for $\mathrm{C}_{13} \mathrm{H}_{21} \mathrm{NO}_{3} \mathrm{~S}(\mathrm{M}+\mathrm{Na})$

Calcd. 294.1134. Found 294.1130.

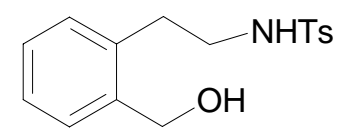

\section{2-Hydroxymethyl-1-phenyethyltolylsulfonamide}

${ }^{1} \mathrm{H}-\mathrm{NMR}\left(\mathrm{CDCl}_{3}, 600 \mathrm{MHz}\right) \delta(\mathrm{ppm})$

${ }^{13} \mathrm{C}-\mathrm{NMR}\left(\mathrm{CDCl}_{3}, 150 \mathrm{MHz}\right) \delta(\mathrm{ppm})$

MS (ESI-) m/z (\%) for $\mathrm{C}_{16} \mathrm{H}_{18} \mathrm{NO}_{3} \mathrm{~S}(\mathrm{M}-\mathrm{H})$ $2.44(\mathrm{~s}, 3 \mathrm{H}), 2.71(\mathrm{t}, 2 \mathrm{H}), 3.88(\mathrm{t}, 2 \mathrm{H}), 4.07(\mathrm{~s}$, 2H), 7.12 (d, $J=4.0 \mathrm{~Hz}, 2 \mathrm{H}), 7.18(\mathrm{~d}, J=7.6$ $\mathrm{Hz}, 1 \mathrm{H}), 7.25(\mathrm{~m}, 1 \mathrm{H}), 7.31(\mathrm{~d}, J=8.0,2 \mathrm{H})$, $7.78(\mathrm{~d}, J=8.0,2 \mathrm{H})$.

$21.5,34.3,45.3,63.7,126.6,127.3,128.9$, 129.8, 129.9, 134.4, 142.0 .

Calcd. 304.1. Found 304.2 (100\%). 
TsHN

$\mathrm{OH}$

\section{N-Hydroxyethyl-tolylsulfonamide}

${ }^{1} \mathrm{H}-\mathrm{NMR}\left(\mathrm{CDCl}_{3}, 400 \mathrm{MHz}\right) \delta(\mathrm{ppm})$

${ }^{13} \mathrm{C}-\mathrm{NMR}\left(\mathrm{CDCl}_{3}, 100 \mathrm{MHz}\right) \delta(\mathrm{ppm})$

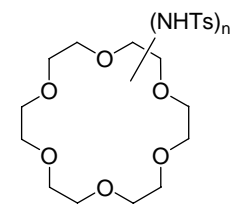

Tosylamine-18-crown-6 (mixture)

${ }^{1} \mathrm{H}-\mathrm{NMR}\left(\mathrm{CDCl}_{3}, 400 \mathrm{MHz}\right) \delta(\mathrm{ppm})$

MS (ESI-) m/z (\%) (M-H)

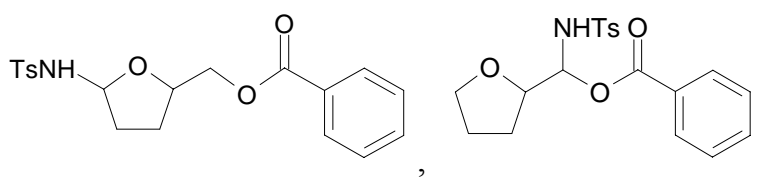

${ }^{1} \mathrm{H}-\mathrm{NMR}\left(\mathrm{CDCl}_{3}, 400 \mathrm{MHz}\right) \delta(\mathrm{ppm})$

${ }^{13} \mathrm{C}-\mathrm{NMR}-\mathrm{DEPT}\left(\mathrm{CDCl}_{3}, 100 \mathrm{MHz}\right) \delta(\mathrm{ppm})$

MS (ESI-) m/z (\%) for $\mathrm{C}_{19} \mathrm{H}_{20} \mathrm{NO}_{5} \mathrm{~S}(\mathrm{M}-\mathrm{H})$ $2.43(\mathrm{~s}, 3 \mathrm{H}), 3.09(\mathrm{q}, 2 \mathrm{H}), 3.69(\mathrm{t}, 2 \mathrm{H}), 5.20(\mathrm{br}$, $1 \mathrm{H}), 7.31(\mathrm{~d}, J=8.0 \mathrm{~Hz}, 2 \mathrm{H}), 7.76(\mathrm{~d}, J=8.0$ $\mathrm{Hz}, 2 \mathrm{H})$.

21.5, 45.2, 61.3, 127.1, 129.8, 143.6, 144.6.

2.40 (ns, 3nH), 3.25 3.85 (m, nH), $4.72(\mathrm{~d}, J=$ $8.0, \mathrm{nH}), 6.97(\mathrm{~m}, \mathrm{nH}), 7.21 \sim 7.82$ (nd, $J=8.0$ $\sim 8.4 \mathrm{~Hz}, \mathrm{nH})$.

Calcd. $n=1.432 .5 ; n=2,601.7 ; n=3,770.9 ; n=4$, 939.2

Found. 432.6; 601.6; 770.7; 939.5

$1.71 \sim 1.88(\mathrm{~m}, 4 \mathrm{H}), 1.97 \sim 2,15(\mathrm{~m}, 4 \mathrm{H}), 2.24$ $\sim 2.28(\mathrm{~m}, 4 \mathrm{H}), 2.31(\mathrm{~s}, 3 \mathrm{H}), 2.22(\mathrm{~s}, 3 \mathrm{H}), 4.11$ $\sim 4.24(\mathrm{~m}, 4 \mathrm{H}), 5.41 \sim 5.54(\mathrm{~m}, 4 \mathrm{H}), 7.08$ (d, $J=$ $8.4 \mathrm{~Hz}, 2 \mathrm{H}), 7.18(\mathrm{~m}, J=8.4,2 \mathrm{H}), 7.43 \sim 7.49(\mathrm{~m}$, 4H), 7.57 7.62 (m, 2H), $7.69(\mathrm{~d}, J=8.4 \mathrm{~Hz}, 2 \mathrm{H})$, $7.79(\mathrm{~d}, J=8.4 \mathrm{~Hz}, 2 \mathrm{H}), 7.98(\mathrm{t}, 4 \mathrm{H})$.

21.3, $25.9(-), 26.3(-), 32.5(-), 32.8(-), 66.2(-), 66.4$ (-), 75.8, 77.1, 85.5, 85.7, 126.4, 127.1, 127.2, 128.4, 128.5, 129.3, 129.5, 129.6, 133.1, Calcd. 374.1. Found 374.1 (100\%). 


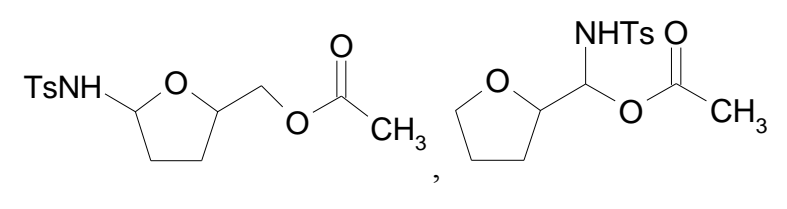

${ }^{1} \mathrm{H}-\mathrm{NMR}\left(\mathrm{CDCl}_{3}, 400 \mathrm{MHz}\right) \delta(\mathrm{ppm})$

MS (ESI-) m/z (\%) for $\mathrm{C}_{14} \mathrm{H}_{18} \mathrm{NO}_{5} \mathrm{~S}(\mathrm{M}-\mathrm{H})$

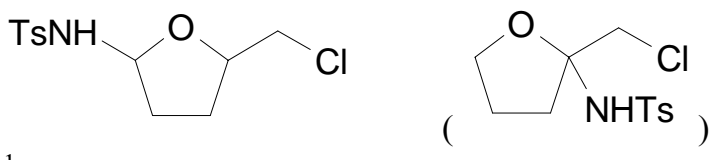

${ }^{1} \mathrm{H}-\mathrm{NMR}\left(\mathrm{CDCl}_{3}, 400 \mathrm{MHz}\right) \delta(\mathrm{ppm})$

MS (ESI+) m/z (\%) for $\mathrm{C}_{12} \mathrm{H}_{17} \mathrm{ClNO}_{3} \mathrm{~S}(\mathrm{M}+\mathrm{H})$

MS (ESI-) m/z (\%) for $\mathrm{C}_{12} \mathrm{H}_{15} \mathrm{ClNO}_{3} \mathrm{~S}(\mathrm{M}-\mathrm{H})$

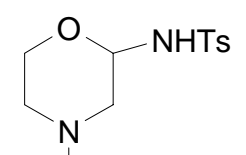

MS (ESI-) m/z (\%) for $\mathrm{C}_{13} \mathrm{H}_{19} \mathrm{~N}_{2} \mathrm{O}_{3} \mathrm{~S}(\mathrm{M}-\mathrm{H})$
$1.62 \sim 2.259(\mathrm{~m}), 2.12(\mathrm{~s}), 2.17(\mathrm{~s}), 2.42(\mathrm{~s}), 2.43(\mathrm{~s})$, 3.84 4.32(m), 5.03 5.12 (m, 2H), 5.32 5.43 (m, $2 \mathrm{H})$, $5.59 \sim 5.63(\mathrm{~m}, 2 \mathrm{H}), 7.25(\mathrm{dd}, J=8.4 \mathrm{~Hz}, 8.0 \mathrm{~Hz}, 4 \mathrm{H})$, $7.80(\mathrm{dd}, J=8.4 \mathrm{~Hz}, 8.0 \mathrm{~Hz}, 4 \mathrm{H})$.

Calcd. 312.0. Found 311.9 (45\%).

1.56 2.02 (m), $2.42(\mathrm{~s}), 2.61 \sim 1.71 \sim 4.17(\mathrm{~m}),, 3.33 \sim$

$4.17(\mathrm{~m}), 5.35 \sim 5.61(\mathrm{~m}), 5.95(\mathrm{~d}, \mathrm{~J}=9.2 \mathrm{~Hz}), 6.12(\mathrm{~d}$, $\mathrm{J}=8.8 \mathrm{~Hz}), 7.31(\mathrm{~m}), 7.78(\mathrm{~m})$.

Calcd. 290.3. Found 290.2 (100\%).

Calcd. 288.0. Found 288.0 (100\%).

Calcd. 283.1. Found 283.2 (88\%). 
HL-14 H1 COC13 2004-11-8

Pulse Sequence: s2pul

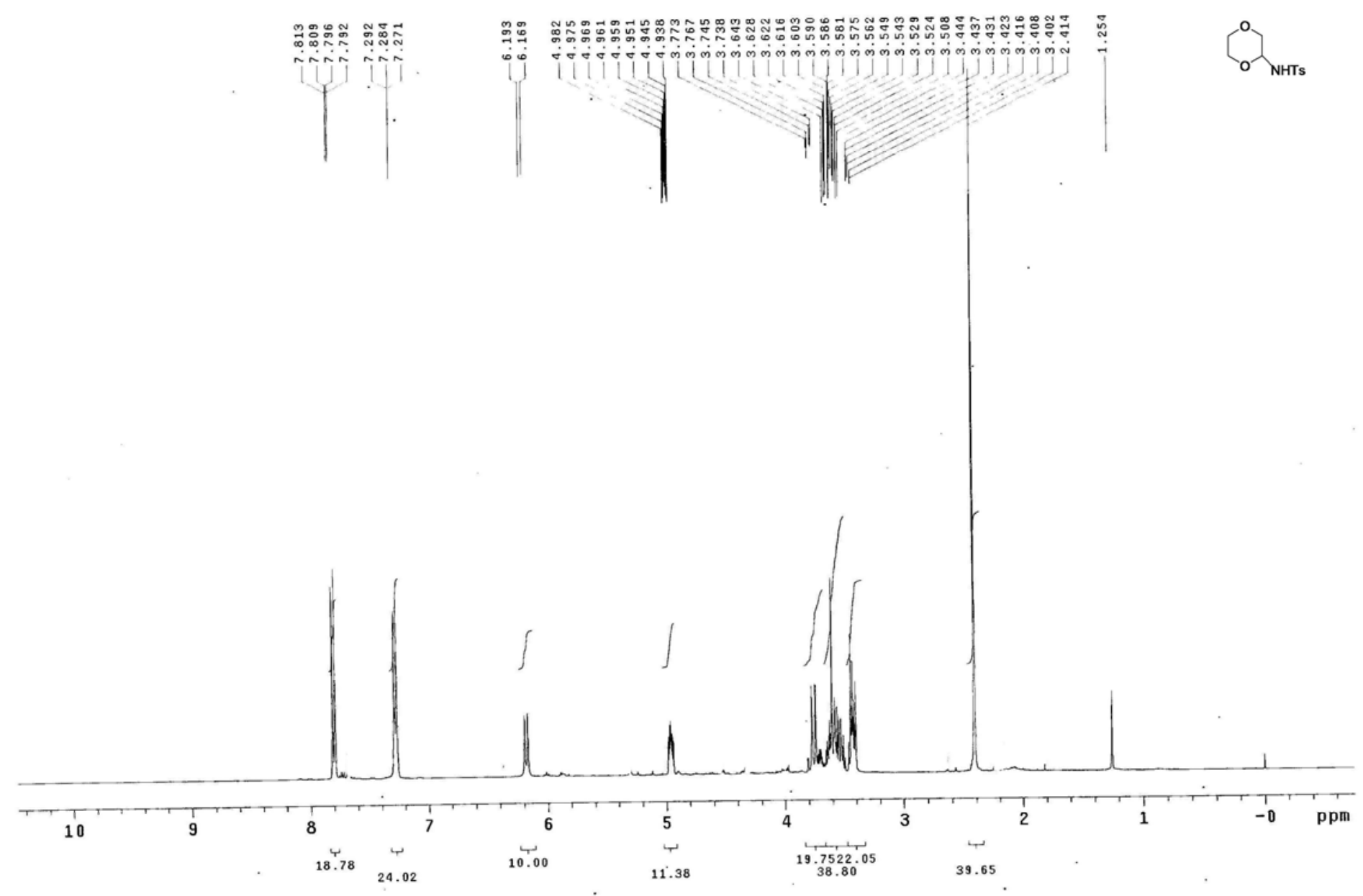


HL-17 H1 $\operatorname{COC}_{13}$ 2005-3-30

Pulse Sequence: s2pul

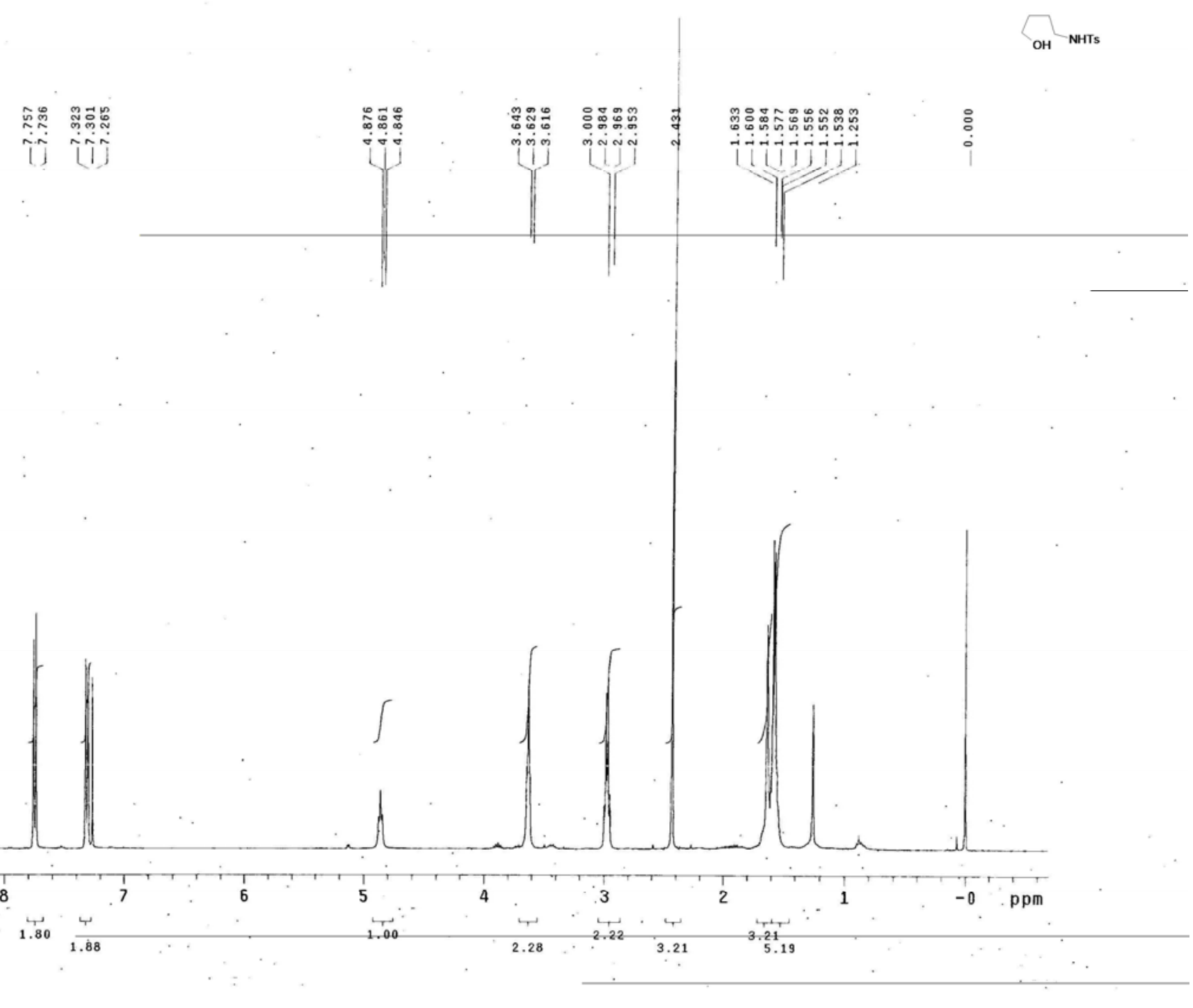




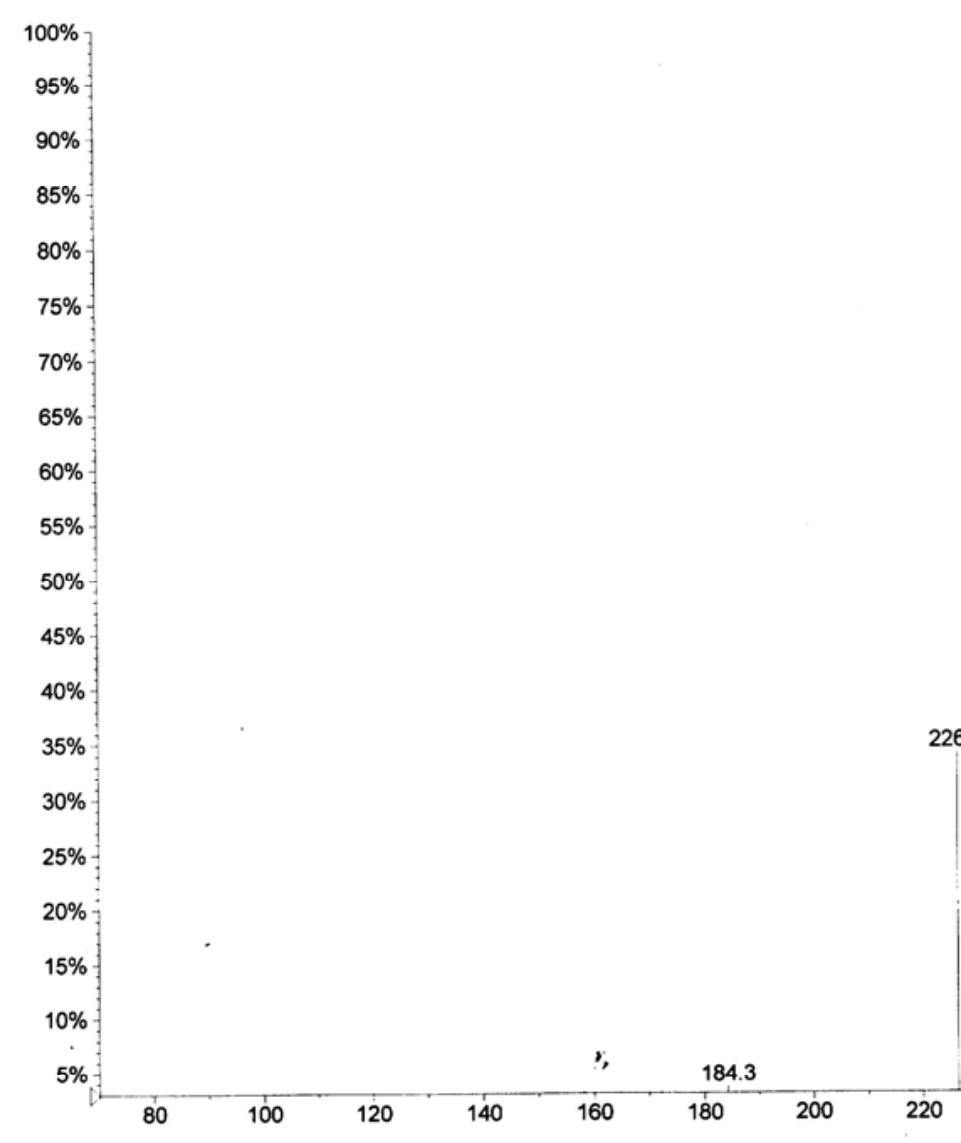

244.4

226.3

261.2

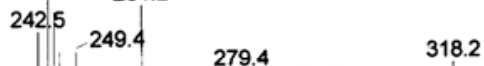
$\mathrm{m} / \mathrm{z}, \mathrm{amu}$ 
HL-18-C-6 H1 $\operatorname{CoC}^{3} \quad 2006-12-20$

Pulse Sequence: s2pul

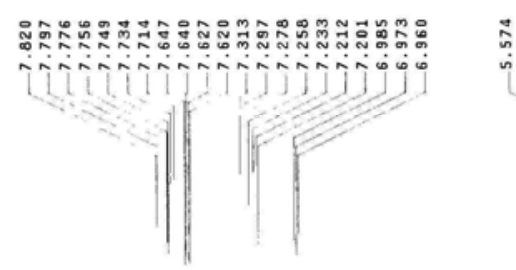

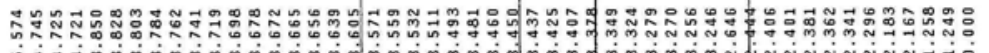

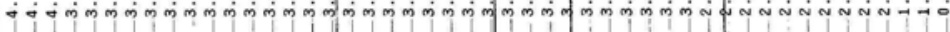

T 1
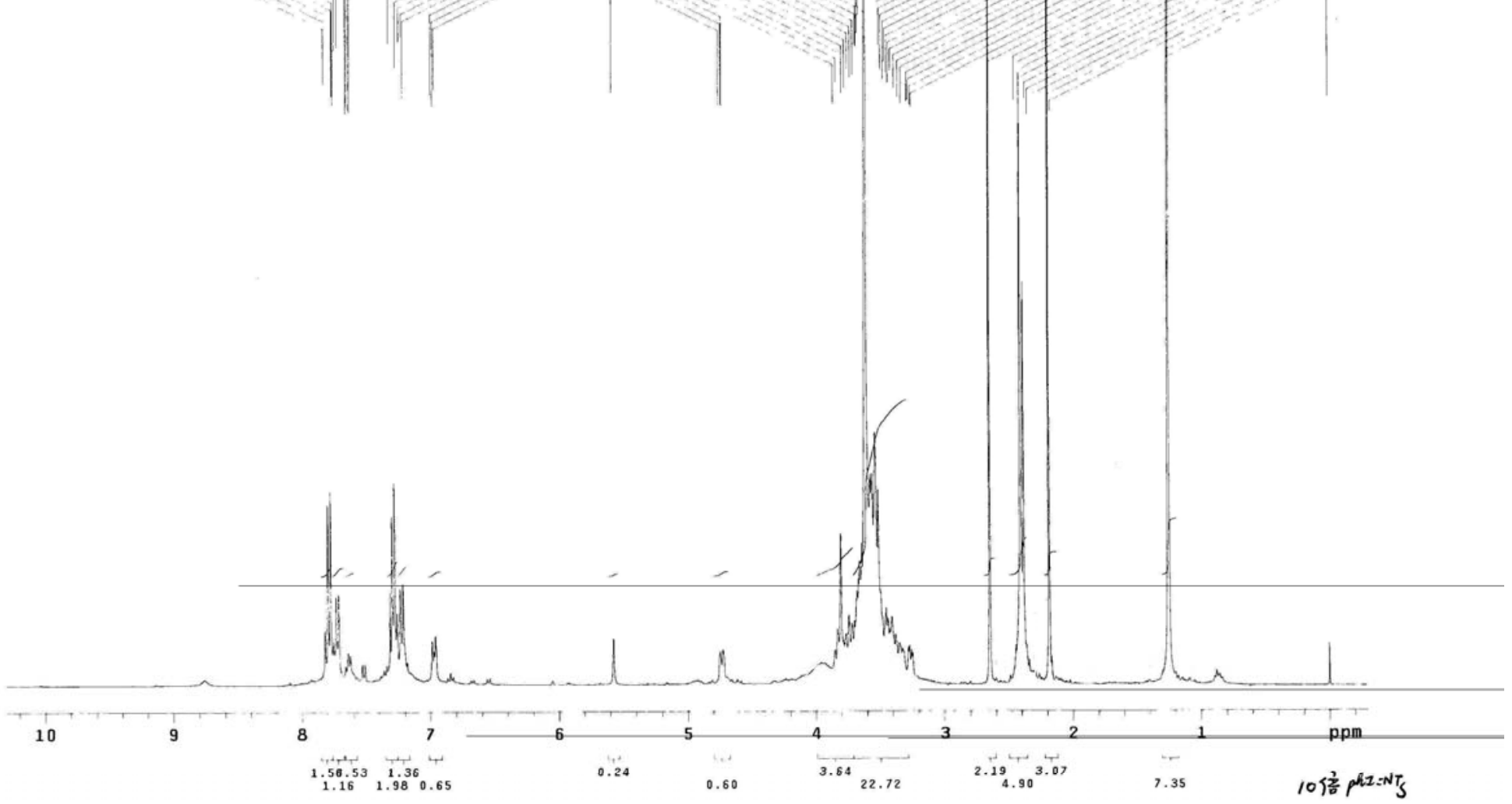
$1.2 \times 1$

- $-\mathrm{Q} 1: 0.033$ to $0.535 \mathrm{~min}$ from Sample 1 (Q1) of qual-061207-HL-15. wiff (Turbo Spray), Centroided

Max. $6.3 \mathrm{e} 5 \mathrm{cps}$.

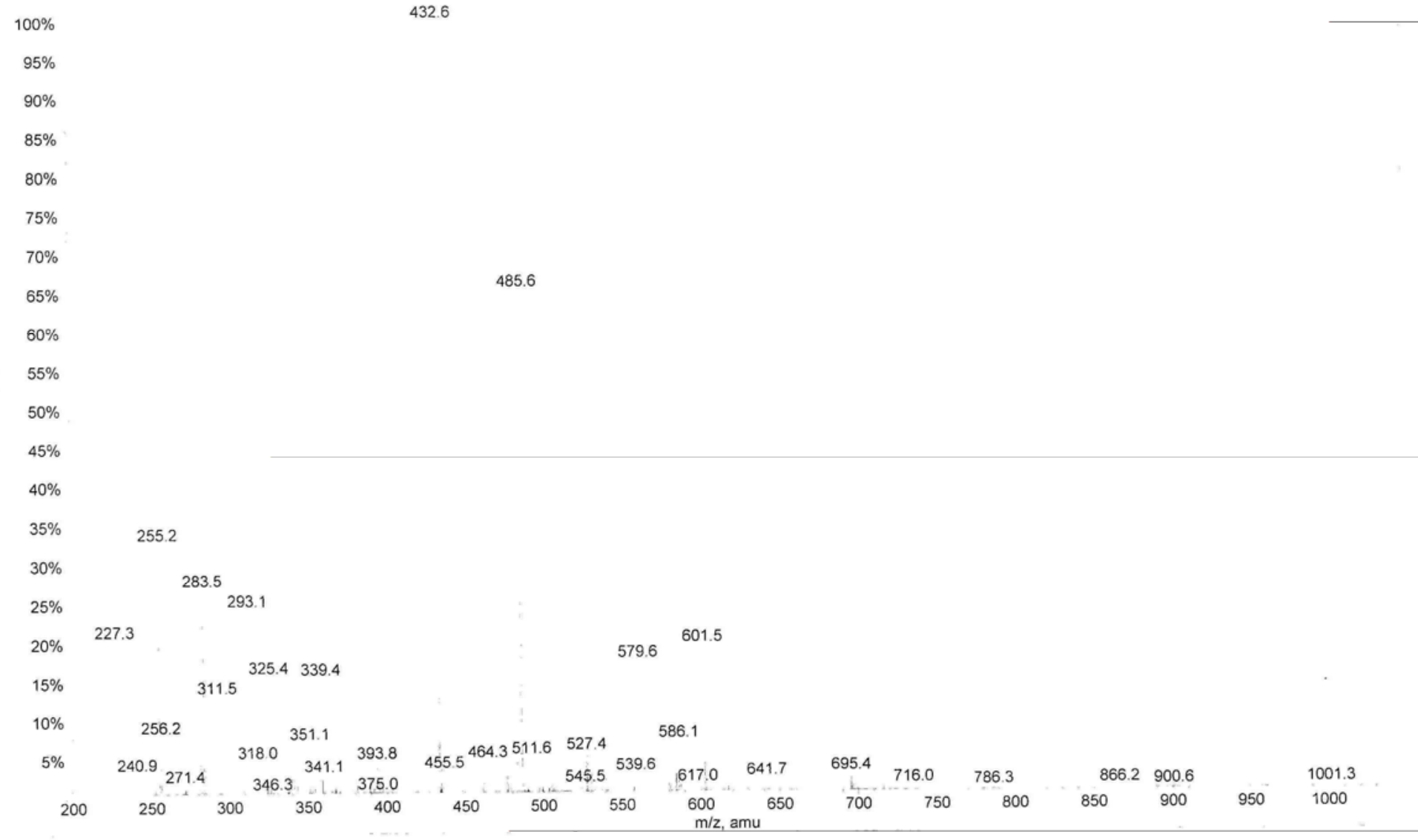


- Q1: 0.033 to $0.602 \mathrm{~min}$ from Sample 2 (Q1) of qual-061207-18-C-6-10.wiff (Turbo Spray). Centroided

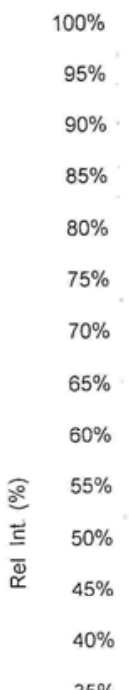

$35 \%$

$30 \%$

$25 \%$

$20 \%$

$15 \%$

$10 \%$

$5 \%$

337.1

351.2

316.1

406.4

256.2

321.0

4326 $\therefore \cdot{ }_{0}^{-}$NHTs $]_{n}$

770.7

250

300

350

400
468.4

450

550
$561.3 \quad 601.6$

$751.5,789.6$

$600 \quad 650 \quad 700$

750

Max $28 \mathrm{e} 6 \mathrm{cps}$

$800 \quad 850$

900

950

1000

12 
- +Q1: 0.000 to 0.635 min from Sample 2 (Q1) of qual-061218-18-C-6. wiff (Turbo Spray). Centroided

$\therefore-\mathrm{NHTs}_{\mathrm{n}}$

Max. $1.5 \mathrm{e} 7 \mathrm{cps}$.

$100 \%$

$95 \%$

$90 \%$

$85 \%$

$80 \%$

$75 \%$

$70 \%$

$65 \%$

ஓ $55 \%$

플 $50 \%$

$45 \%$

$40 \%$

$35 \%$

$30 \%$

$25 \%$

$20 \%$

$15 \%$

$10 \%: 530.2$

$5 \%$

$500 \quad 550$
641.2

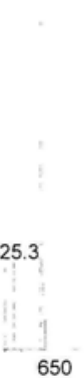

1093.3

072.3

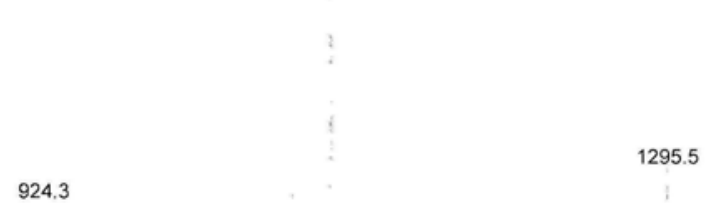

944.5

$1051.6 \quad \begin{array}{cc}1077.4 & 11126.4\end{array}$

1262.1

755.4

734.4
$\quad 824.3$

905.5

$700 \quad 750$

$\frac{1}{4}+\frac{1}{2}$

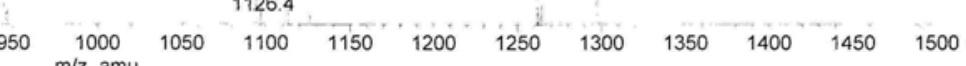


HL-21-2 H1 CDC13 2006-12-20

Pulse Sequence: s2pul

$$
\text { NHro }
$$

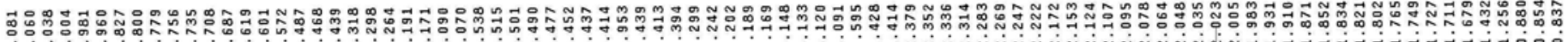

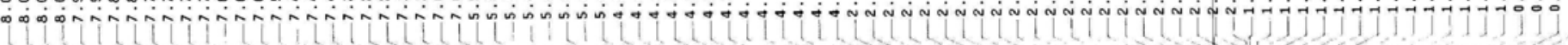
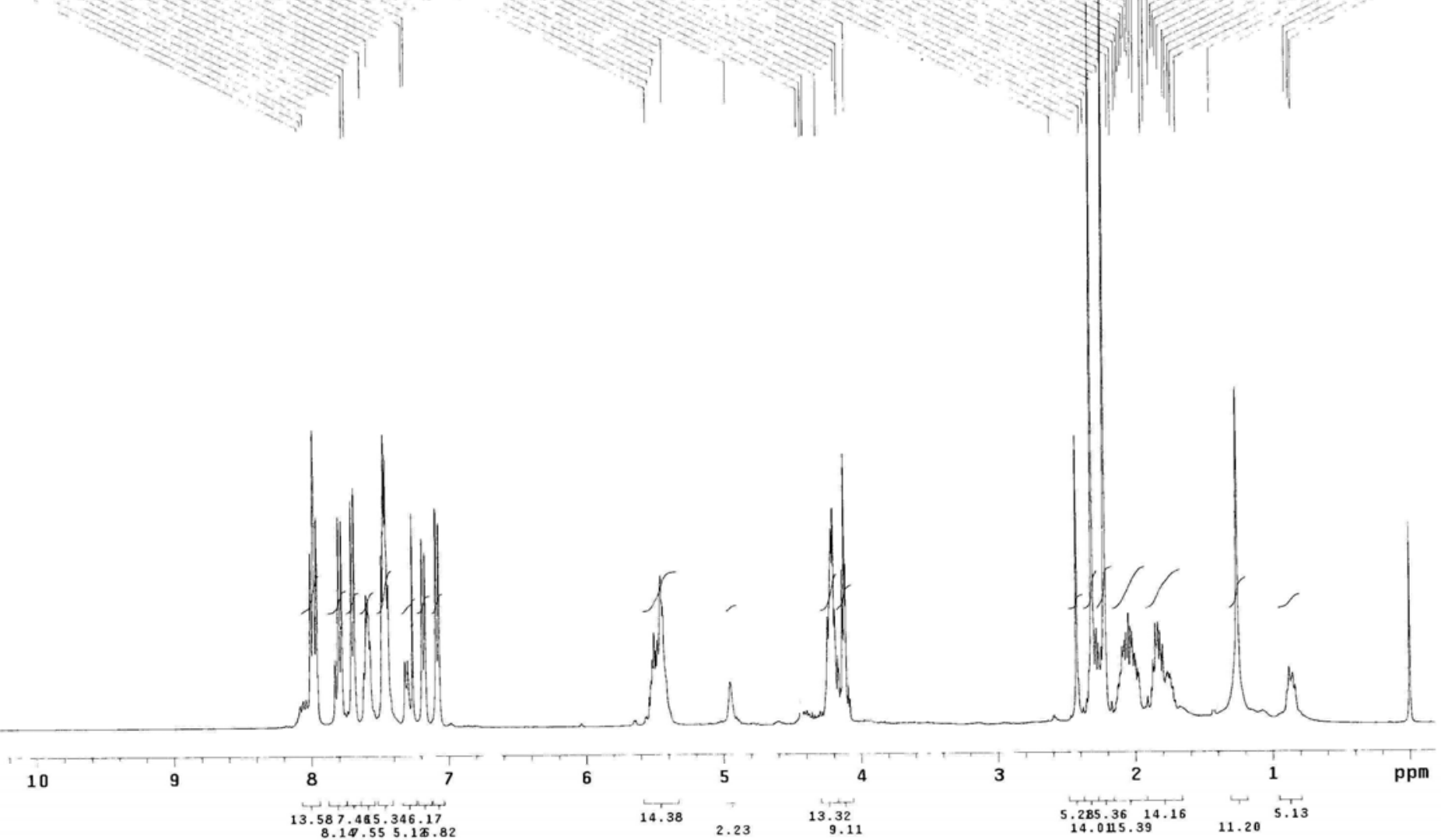
- Q1: 0.067 to $0.635 \mathrm{~min}$ from Sample 2 (Q1) of qual-061207-HL-21.wiff (Turbo Spray)
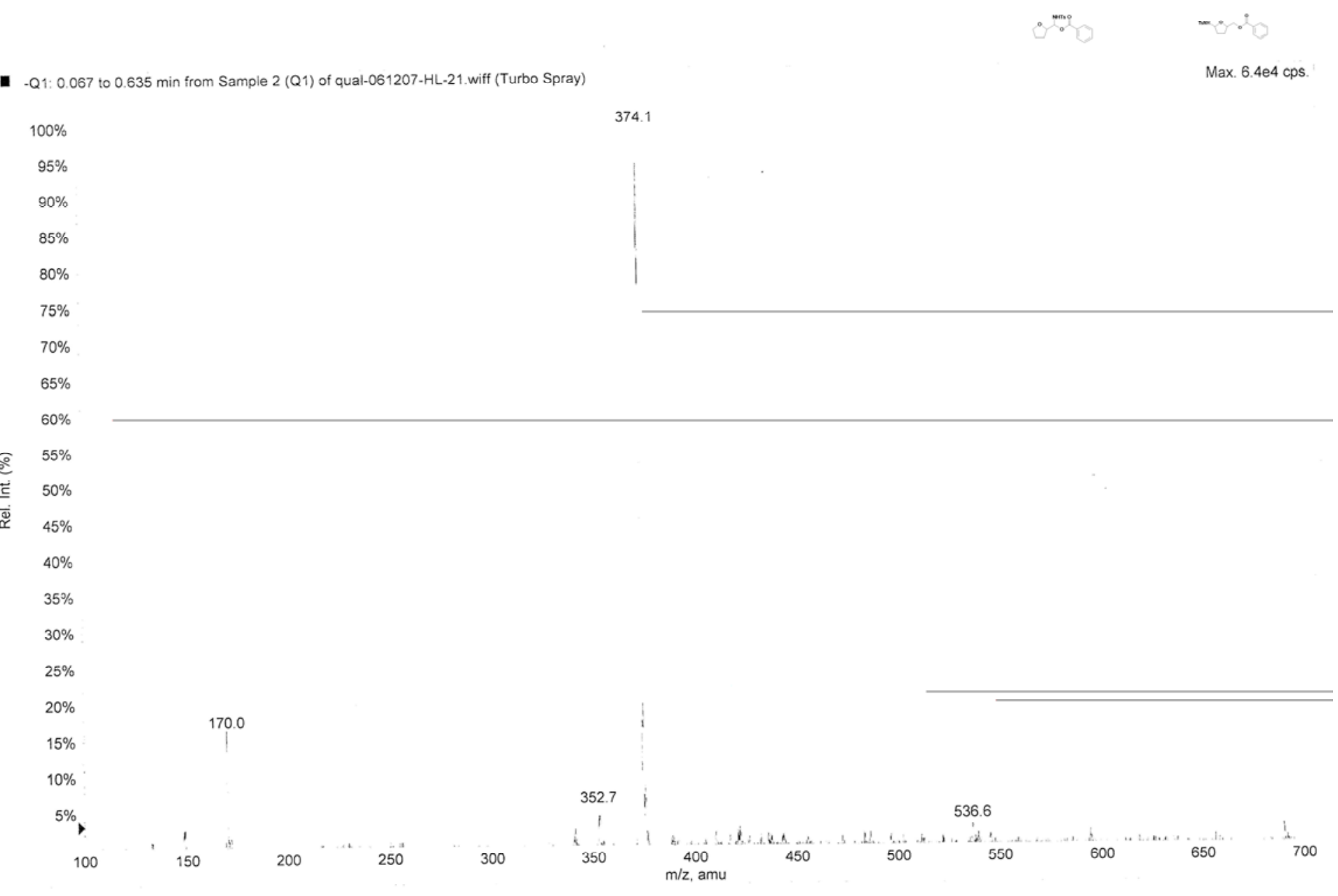
HL-21(12.5) H1 COC13 2006-12-13 Pulse sequence: s2pul

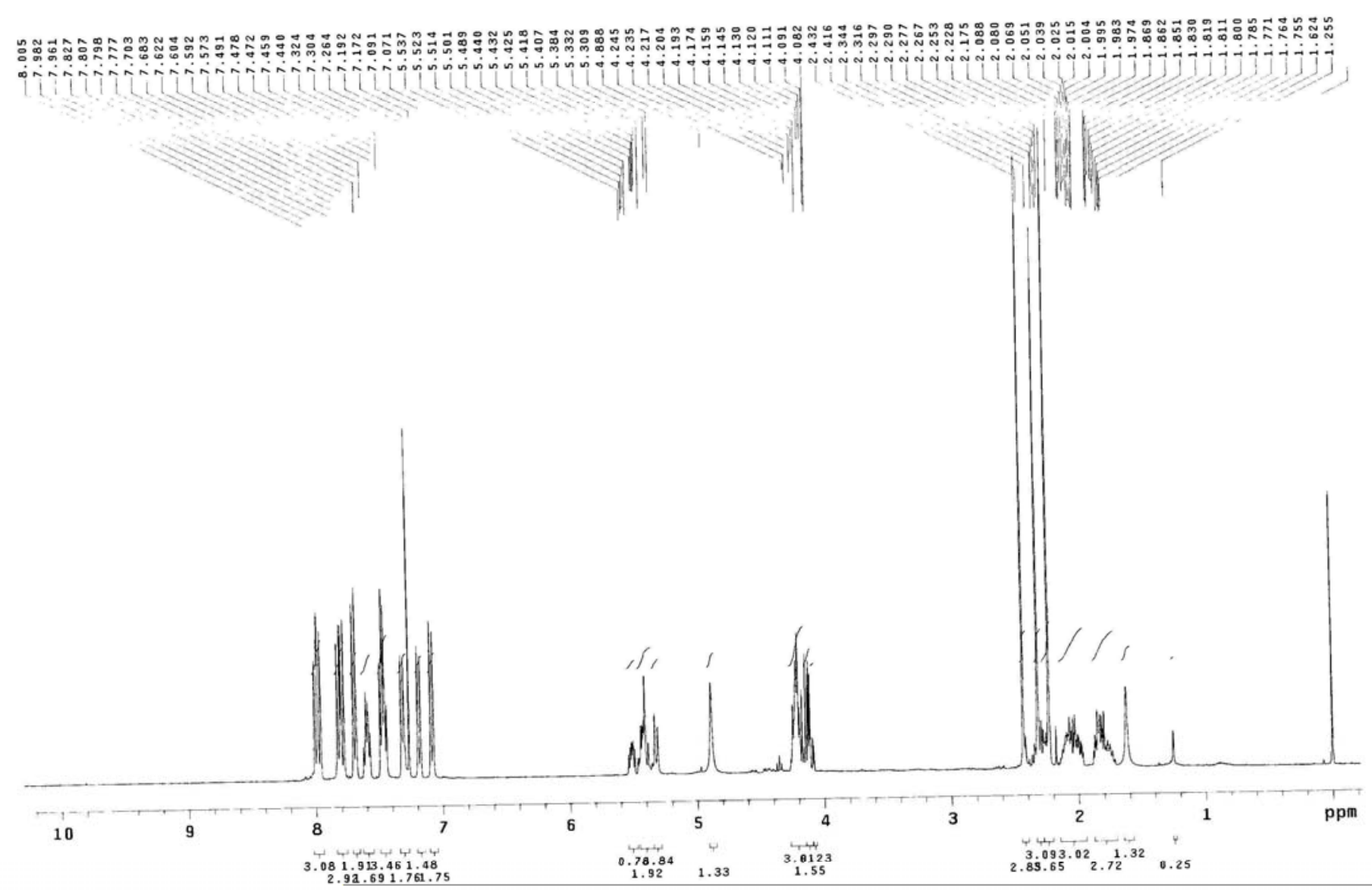


- -Q1: 0067 to 0.635 min from Sample 2 (Q1) of qual-061207-HL-21 wiff (Turbo Spray)

Max. $6.4 \mathrm{e} 4 \mathrm{cps}$

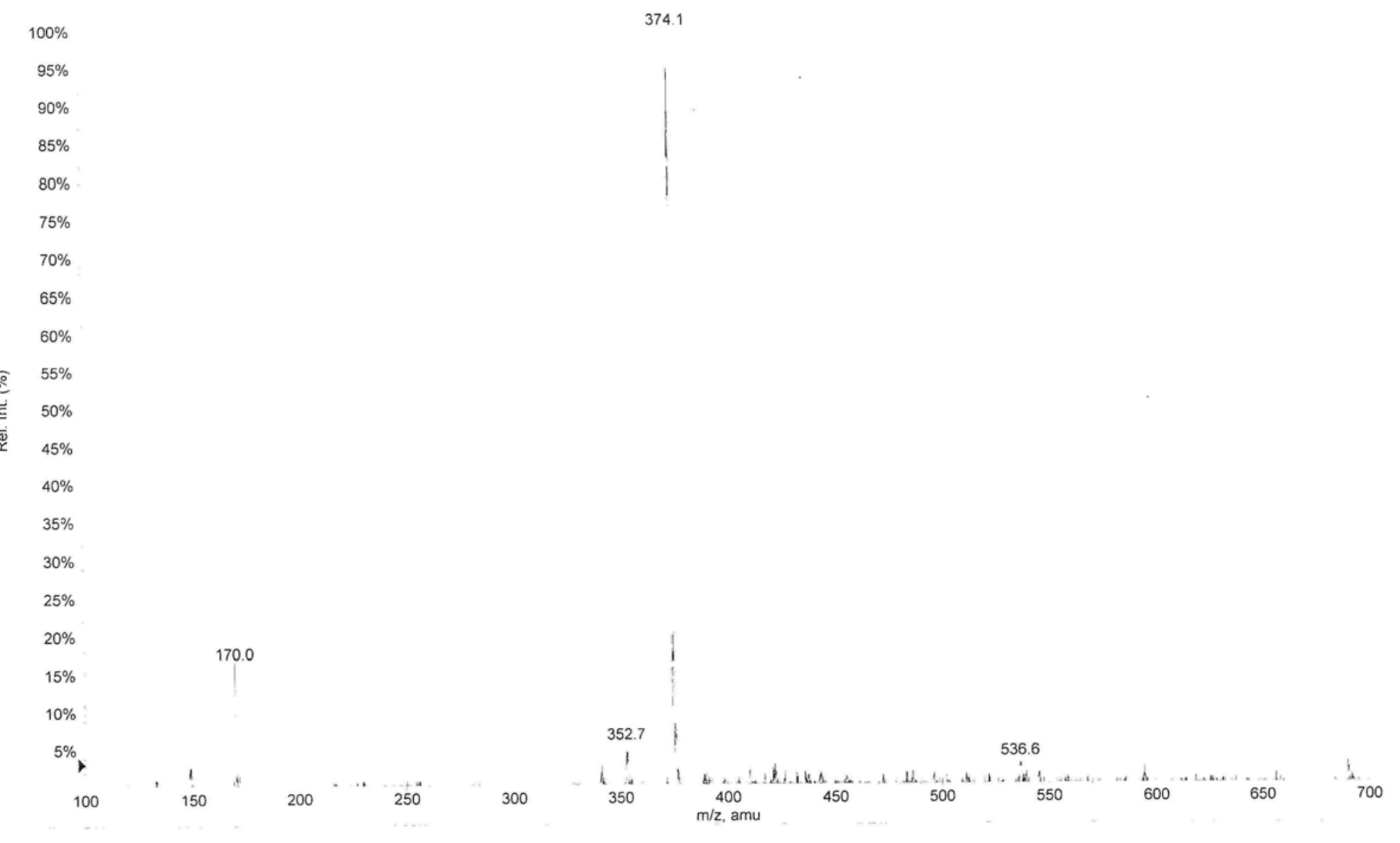


HL-35 H1 COC13 2007-1-31

Pulse Sequence: sâpul
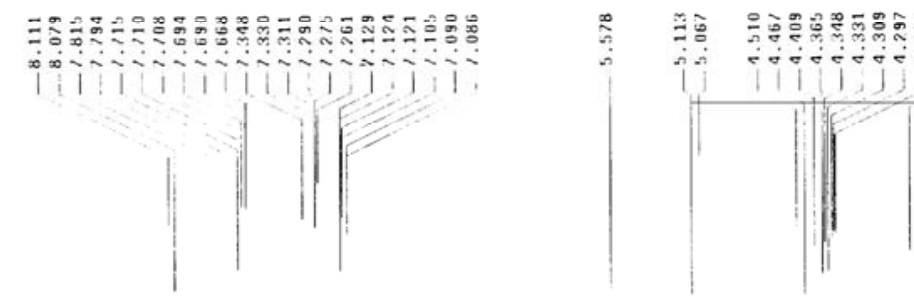

รึำำ ำำ
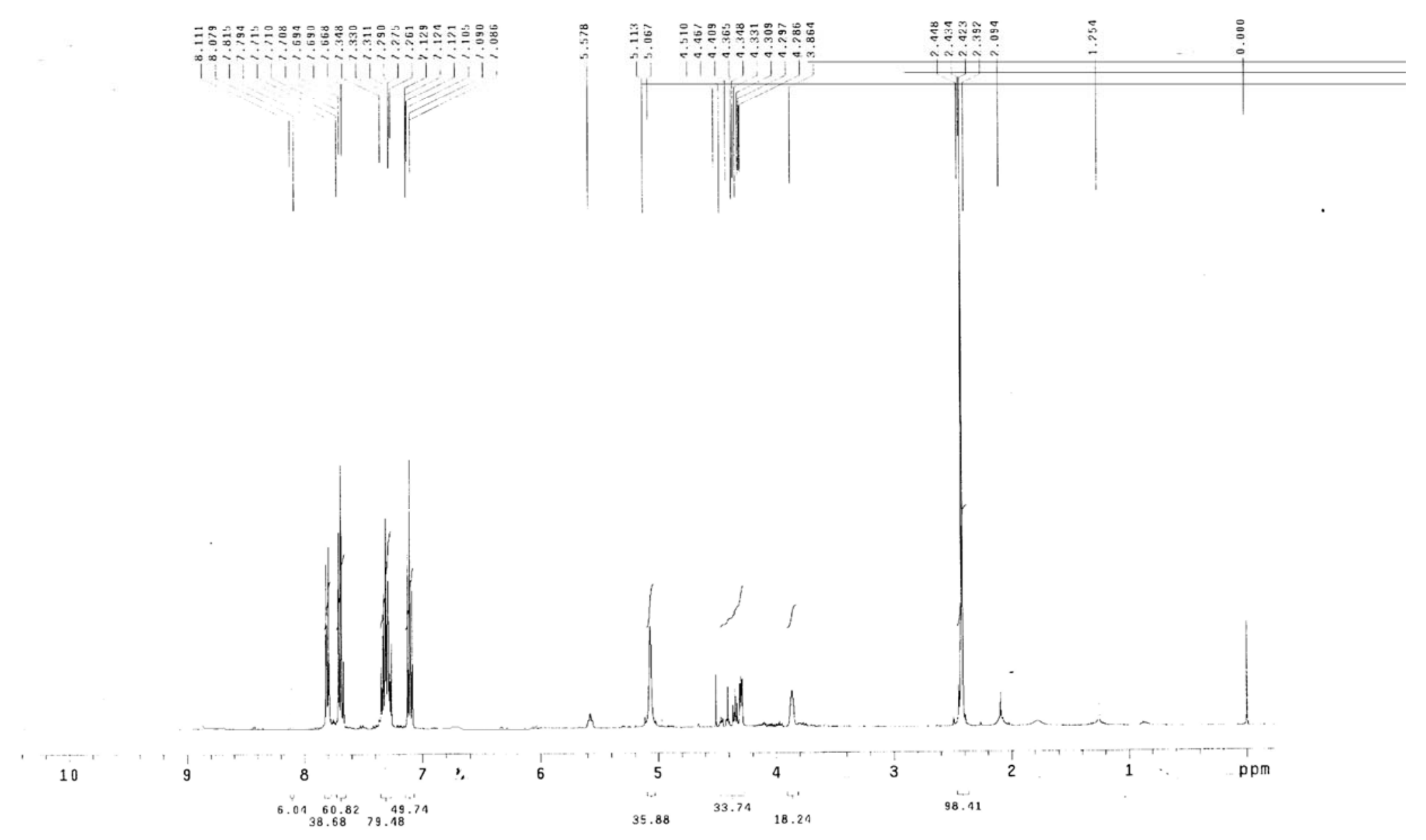


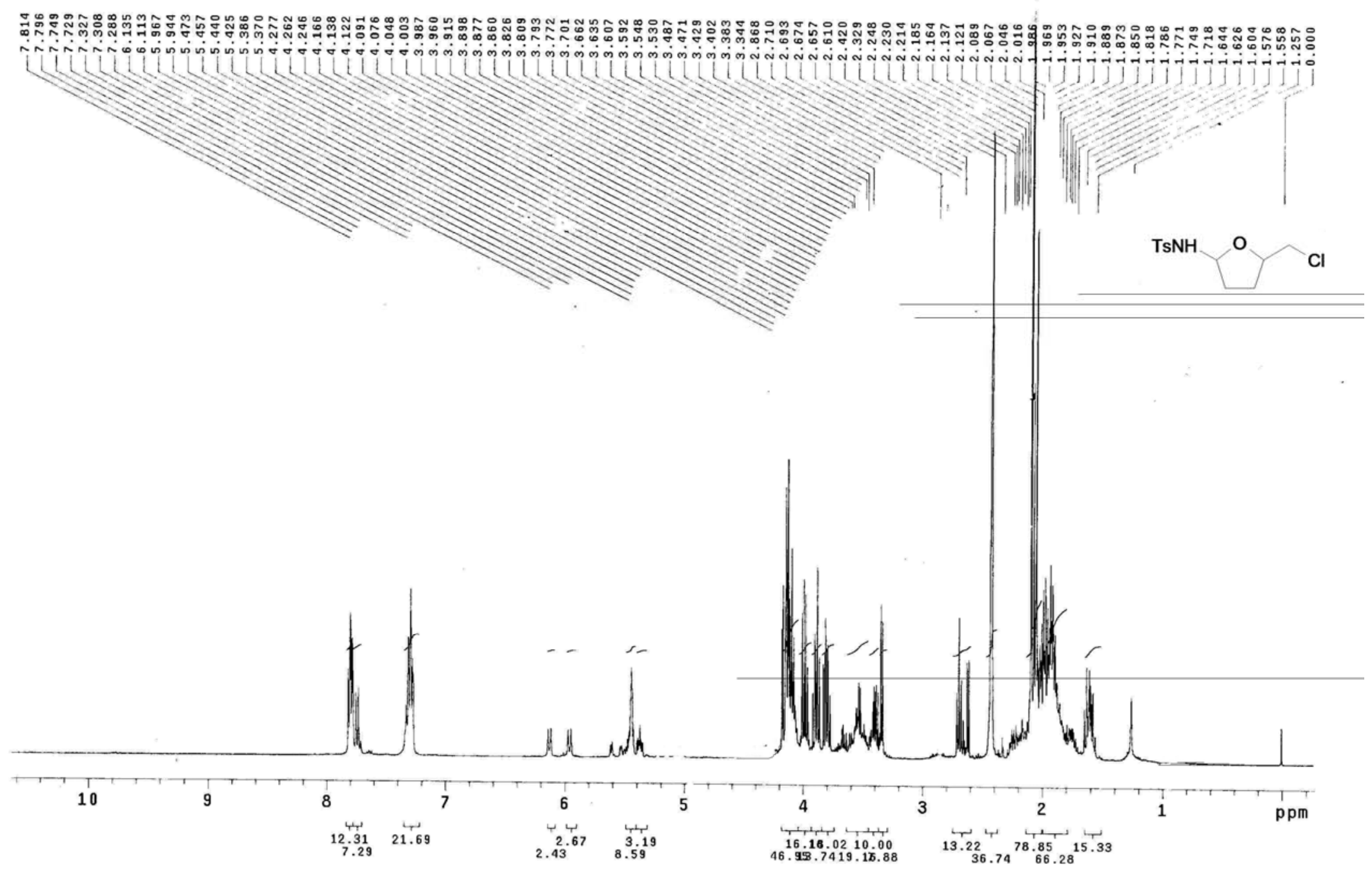




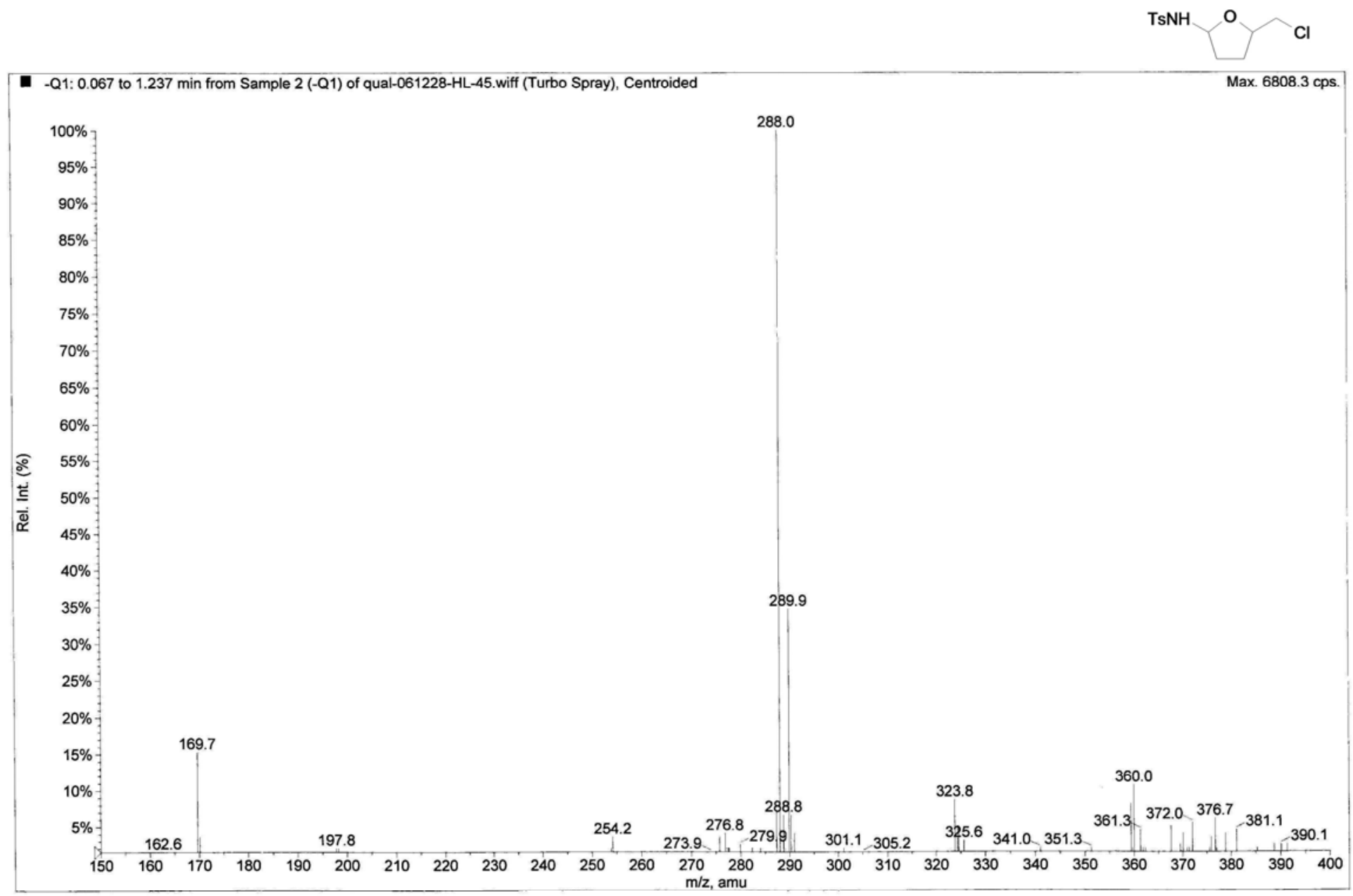




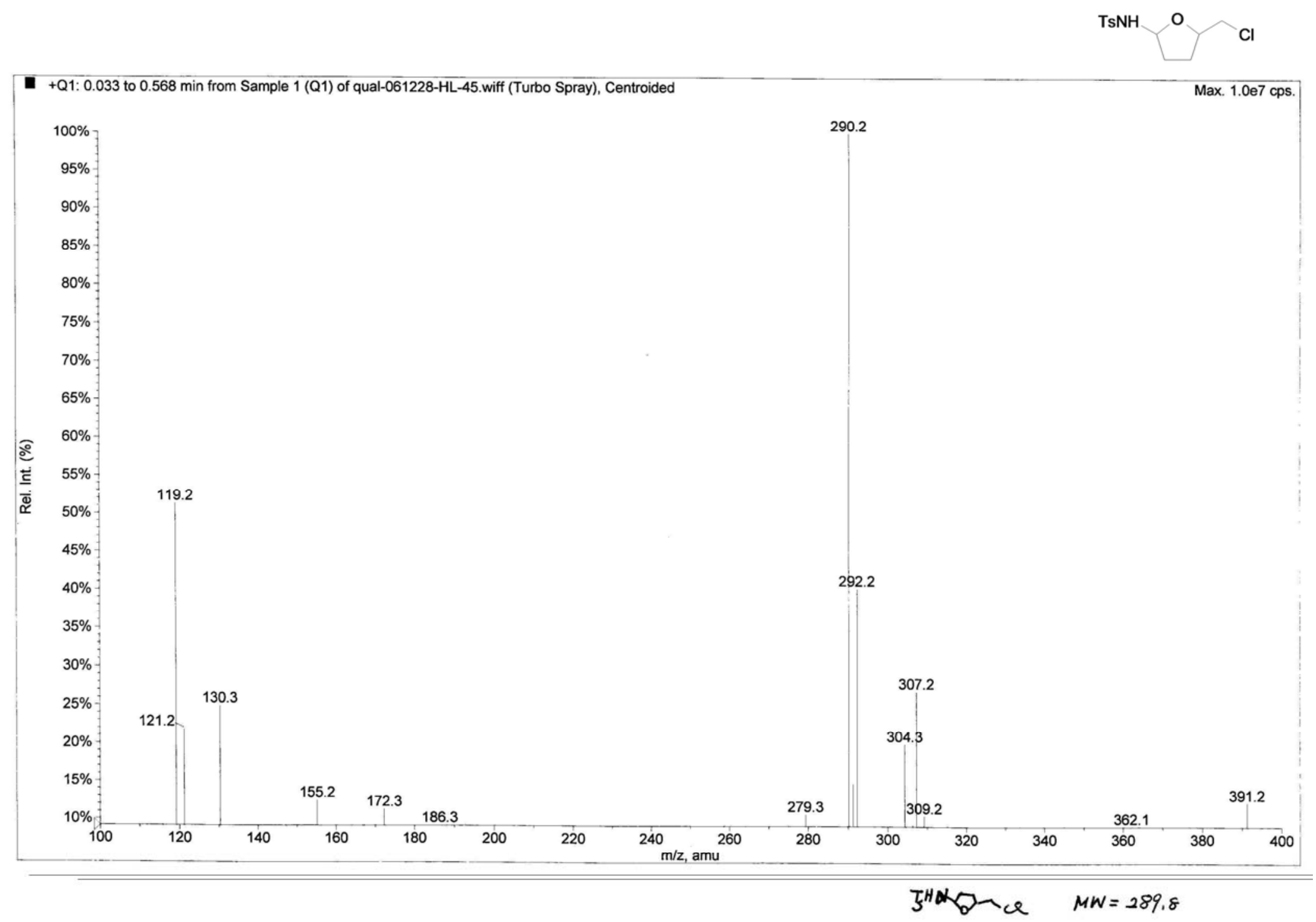


HL-54 H1 CDC13 2006-6-8

Pulse Sequence: s2pul

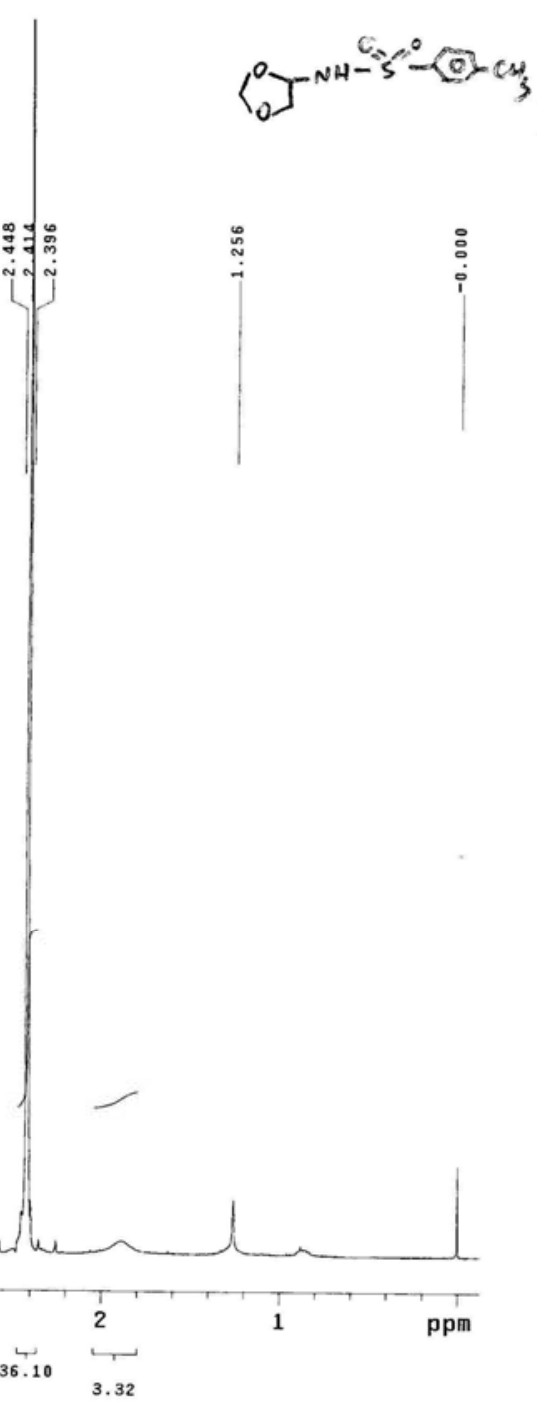


- -Q1: 0.000 to $0.836 \mathrm{~min}$ from Sample 2 (Q1) of qual-061228-HL-81. wiff (Turbo Spray), Centroided

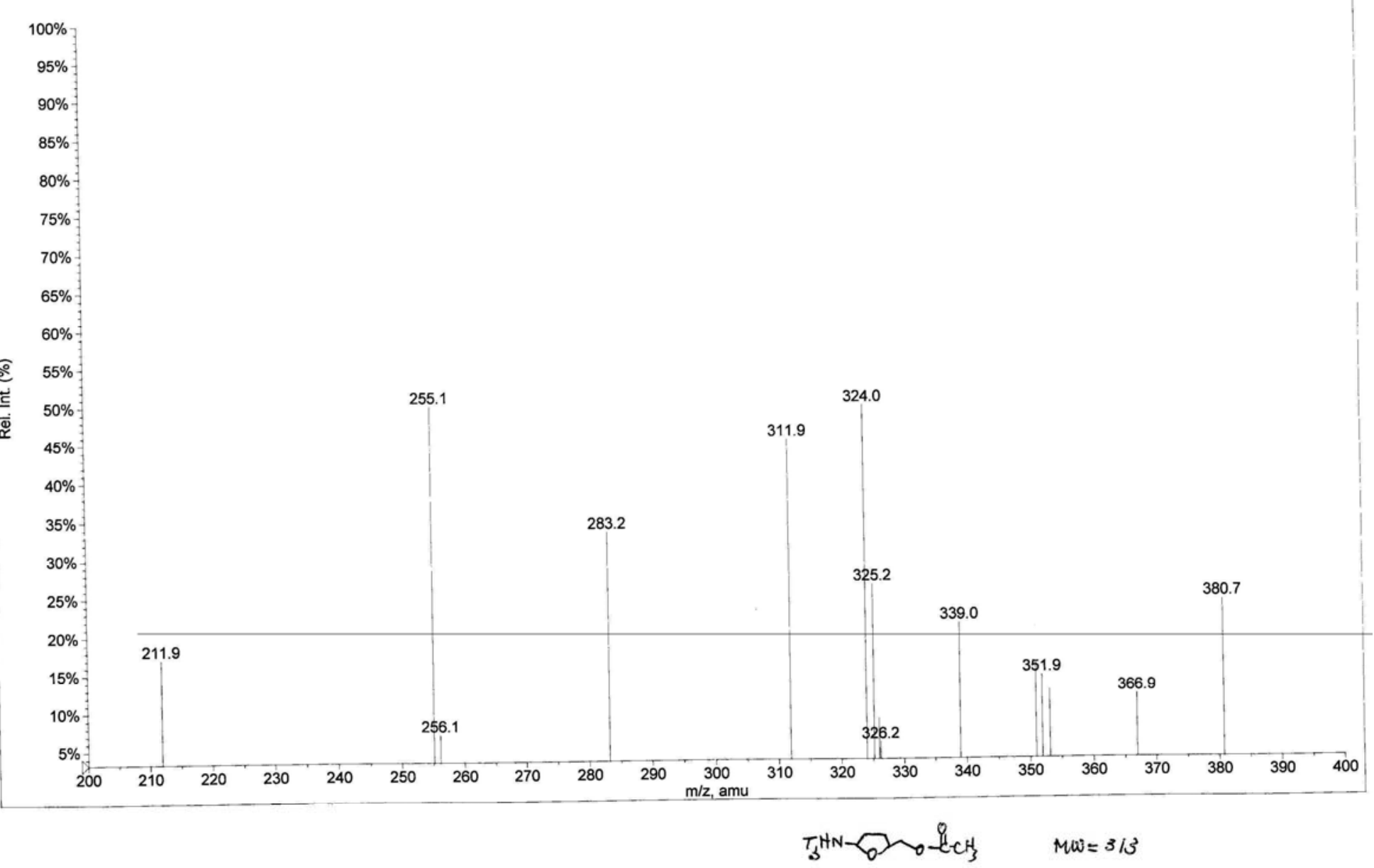




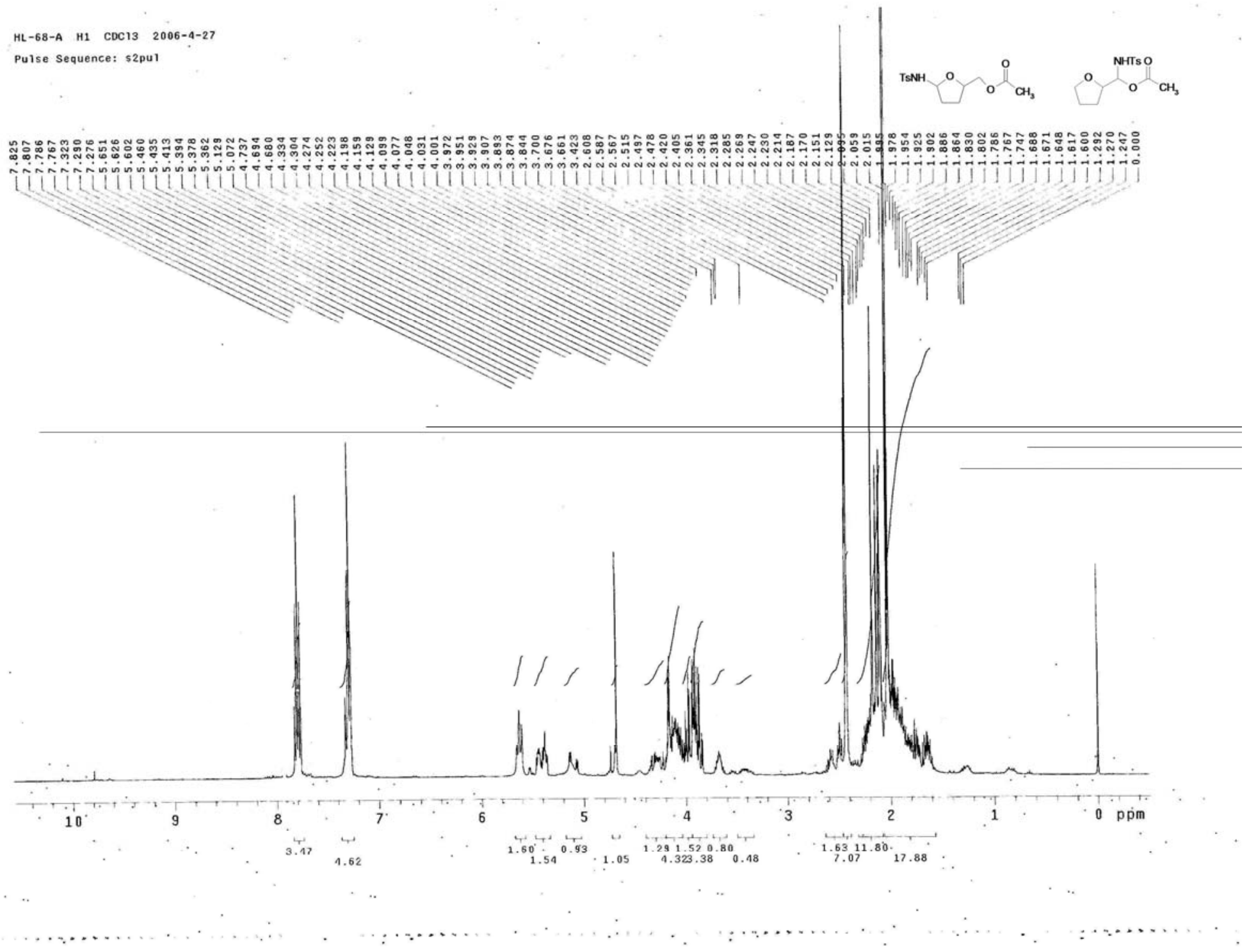


Bruker Avance 600 probe: $13 \mathrm{C}-1 \mathrm{H}$ DUL TE: $300 \mathrm{~K}$ sample: HL70 solvent: CDCL3 spectrum: $1 \mathrm{H}$ पำ

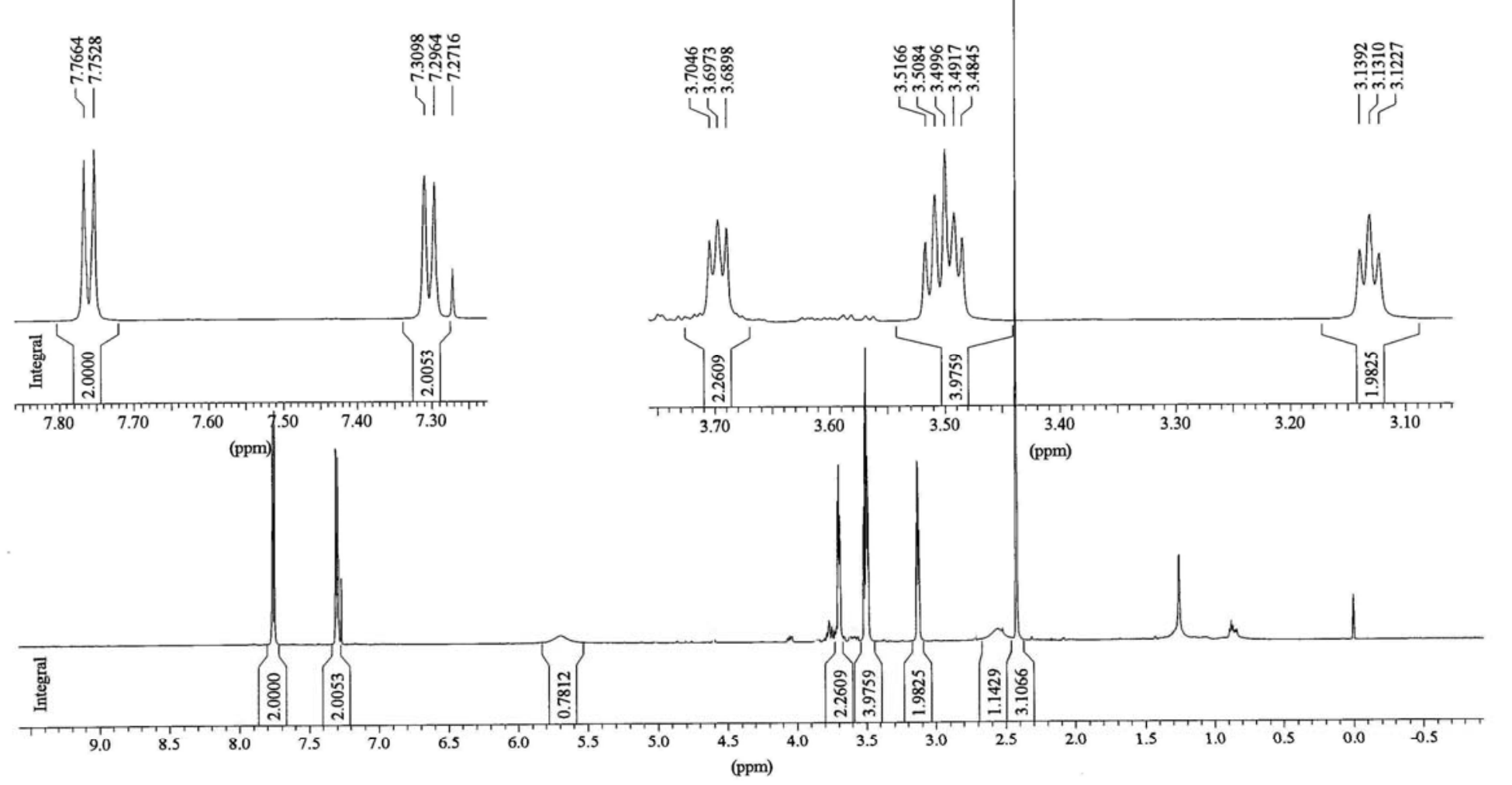




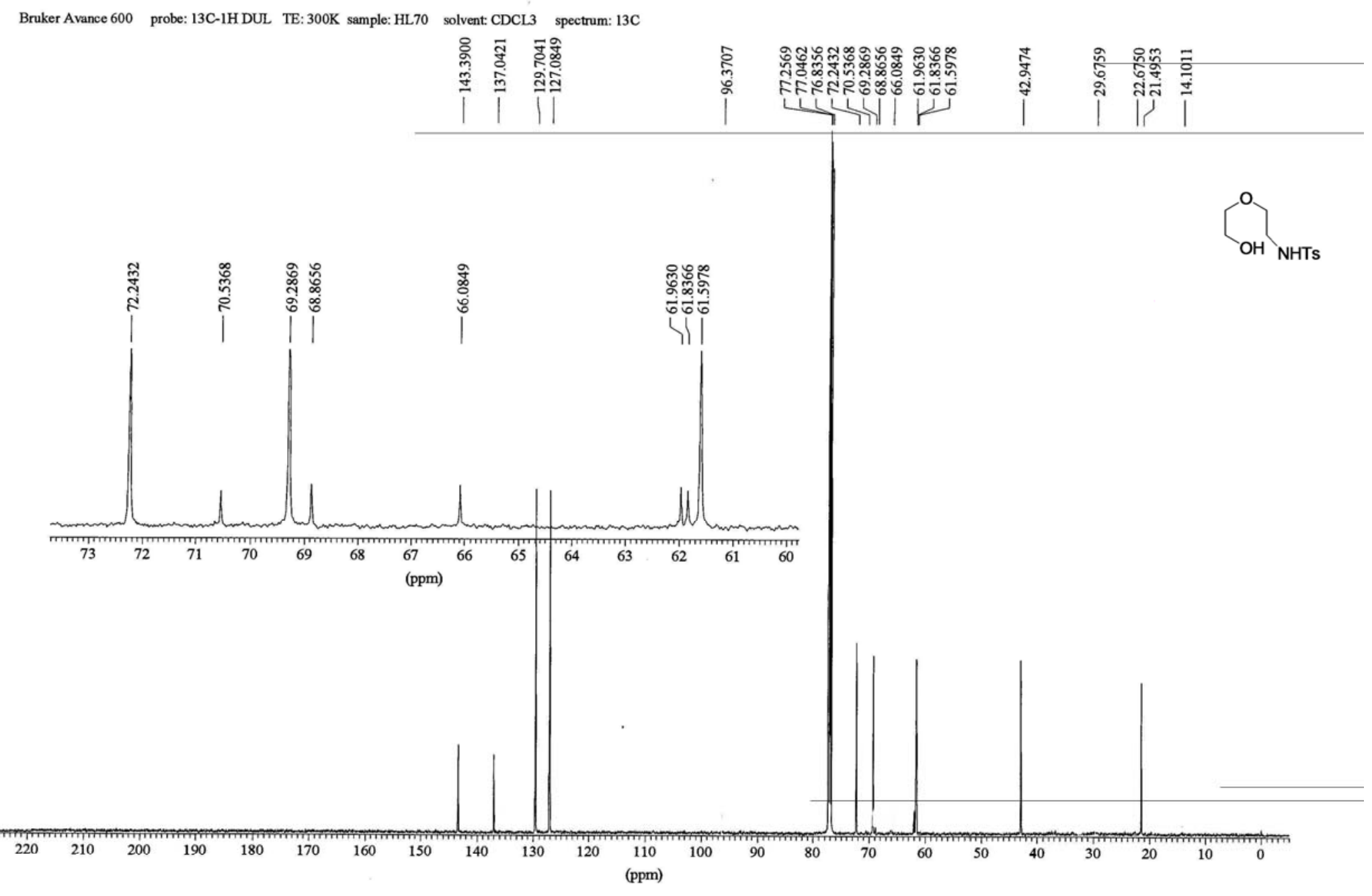




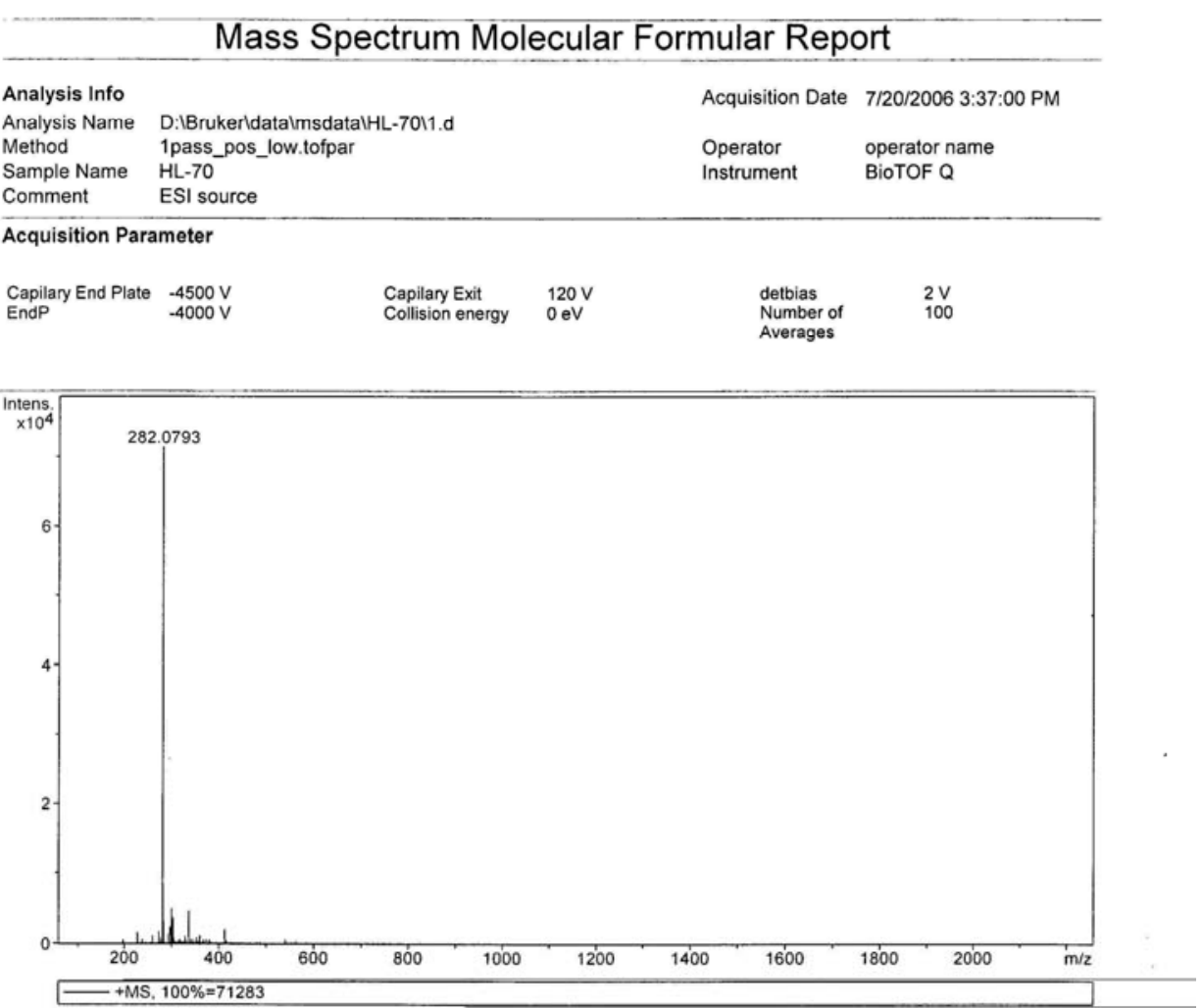

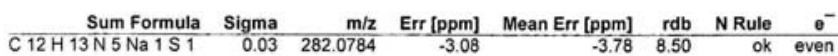

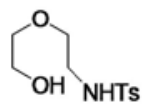




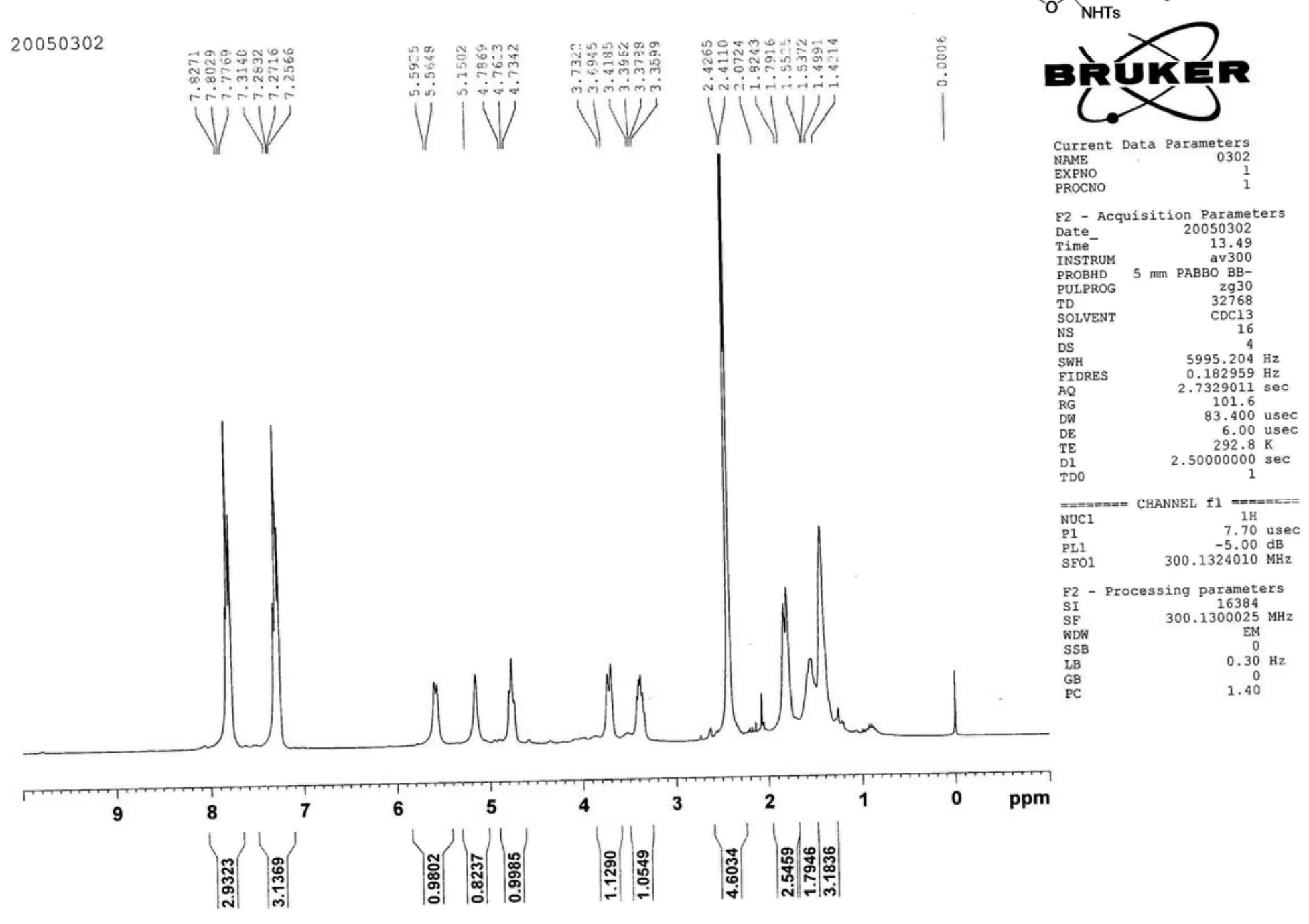




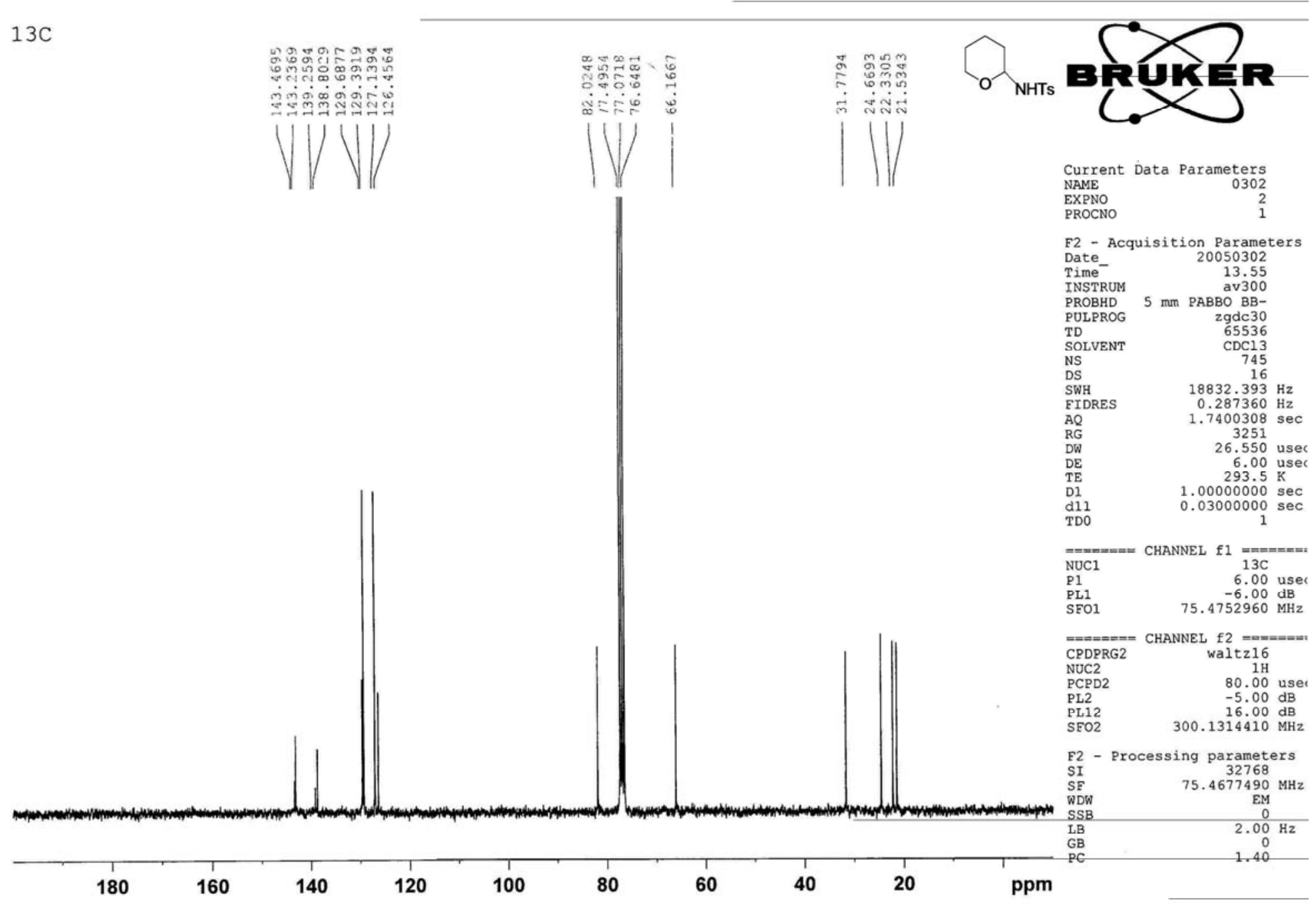




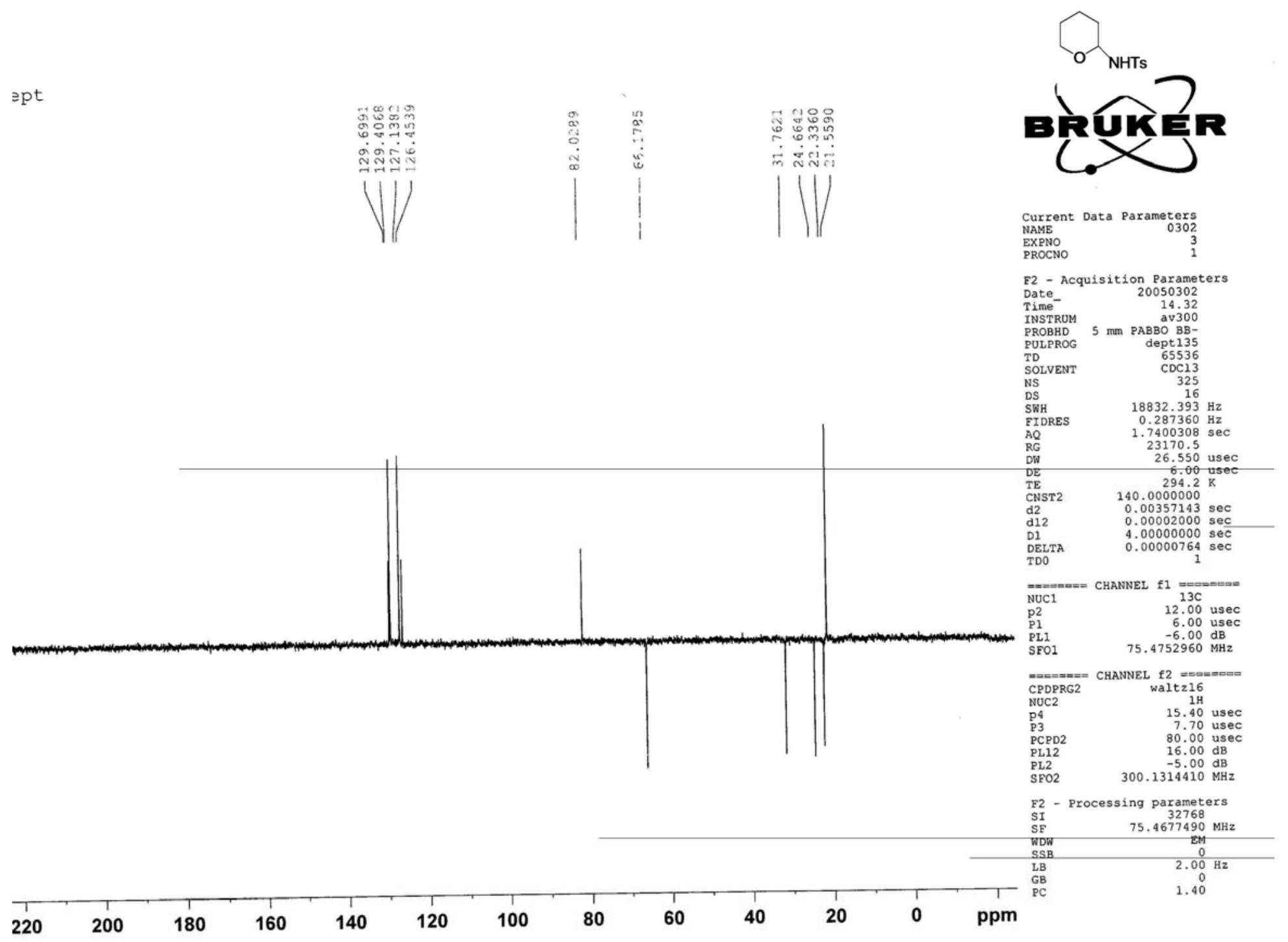




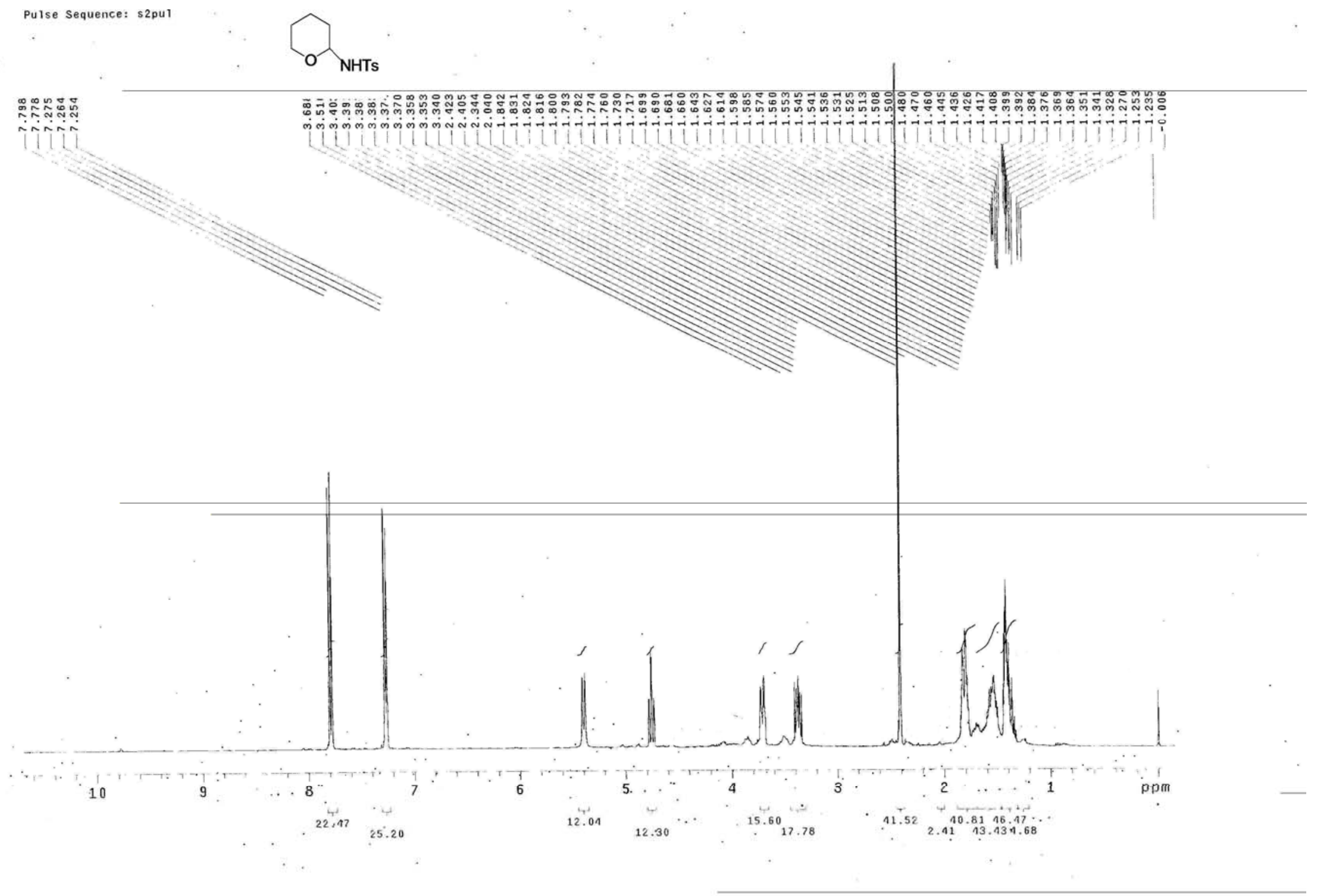




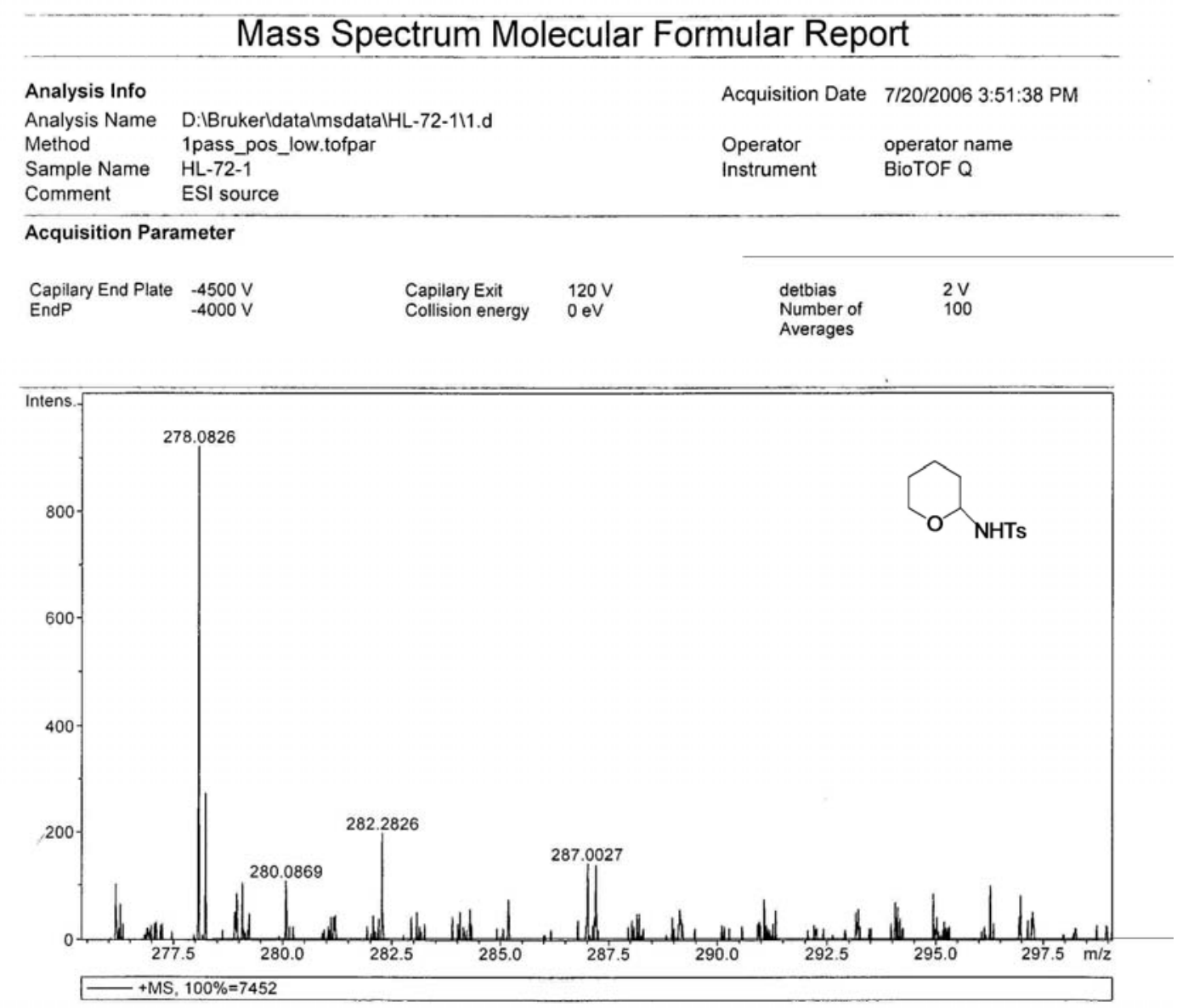

\begin{tabular}{rrrrrrrr} 
Sum Formula & Sigma & $\mathrm{m} / \mathrm{z}$ & Err [ppm] & Mean Err [ppm] & rdb & NRule & $e^{-}$ \\
\hline $\mathrm{C} 12 \mathrm{H} 17 \mathrm{~N} 1 \mathrm{Na} 103 \mathrm{~S} 1$ & 0.04 & 278.0821 & -1.52 & -4.80 & 4.50 & $0 \mathrm{k}$ & even
\end{tabular} 


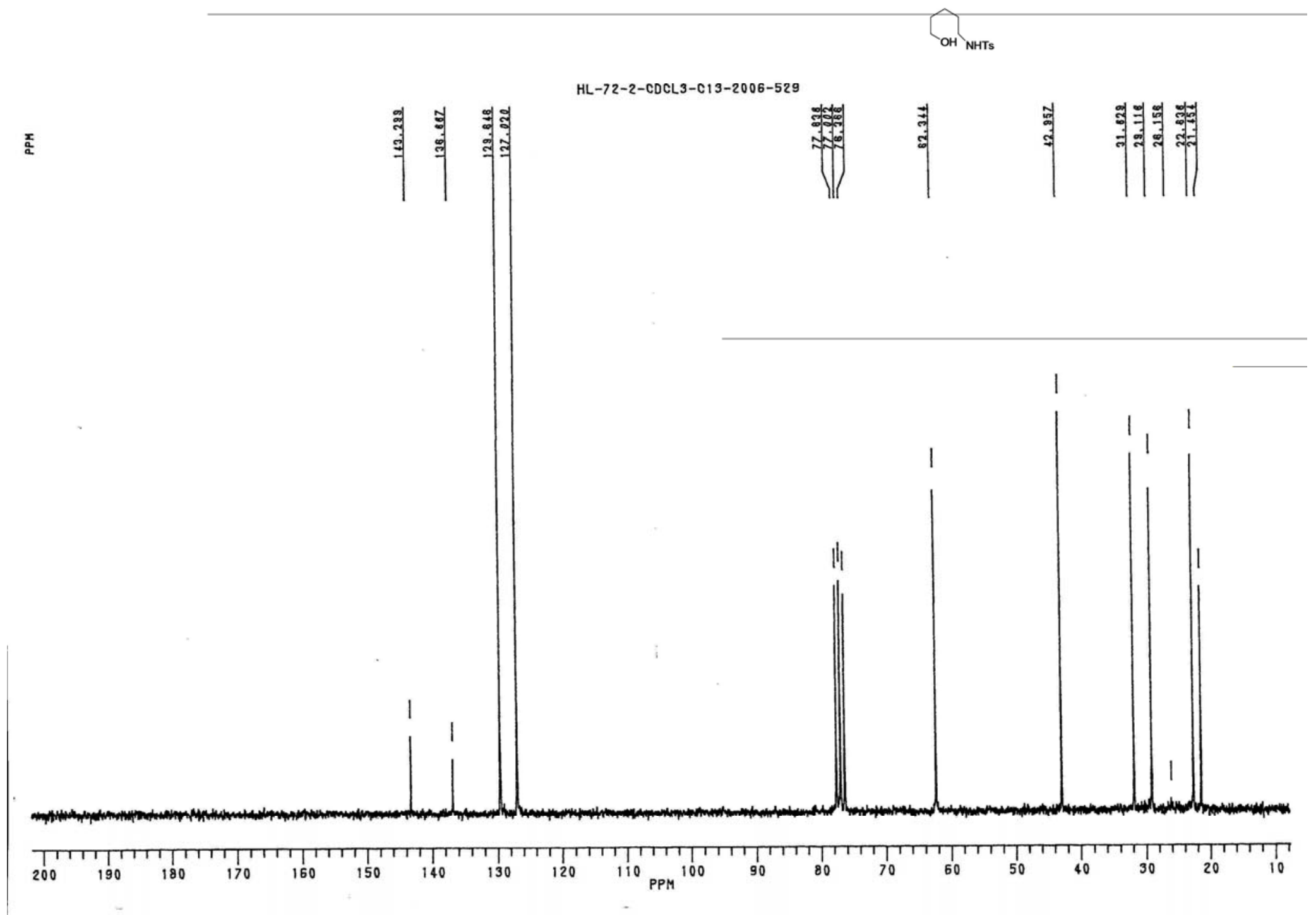


HL-72-2 H1 CDC13 2006-5-26

Pulse Sequence: s2pul

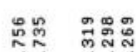

iิ $\tilde{i}^{-1}$

îj

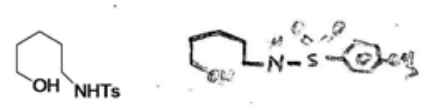

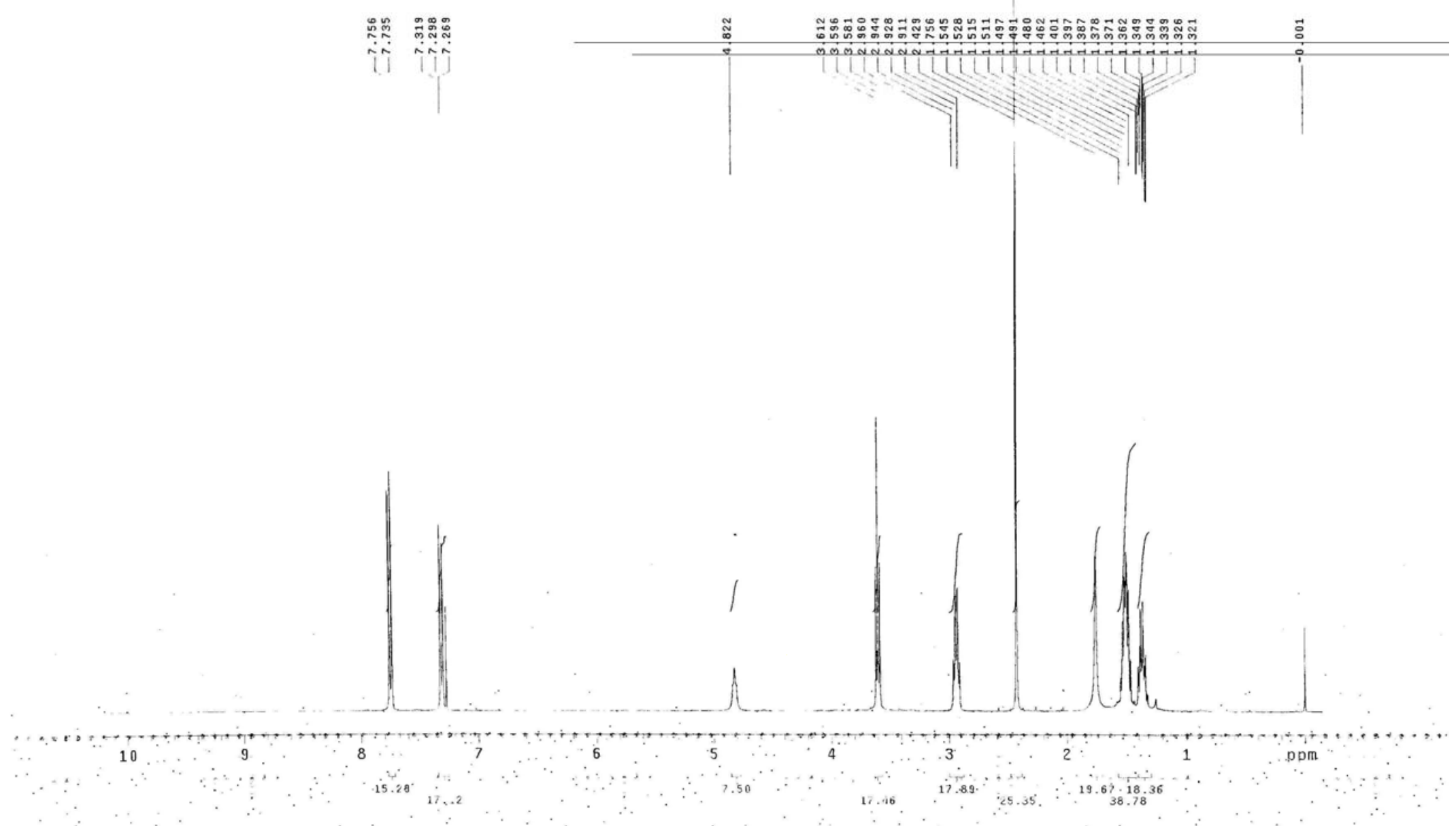




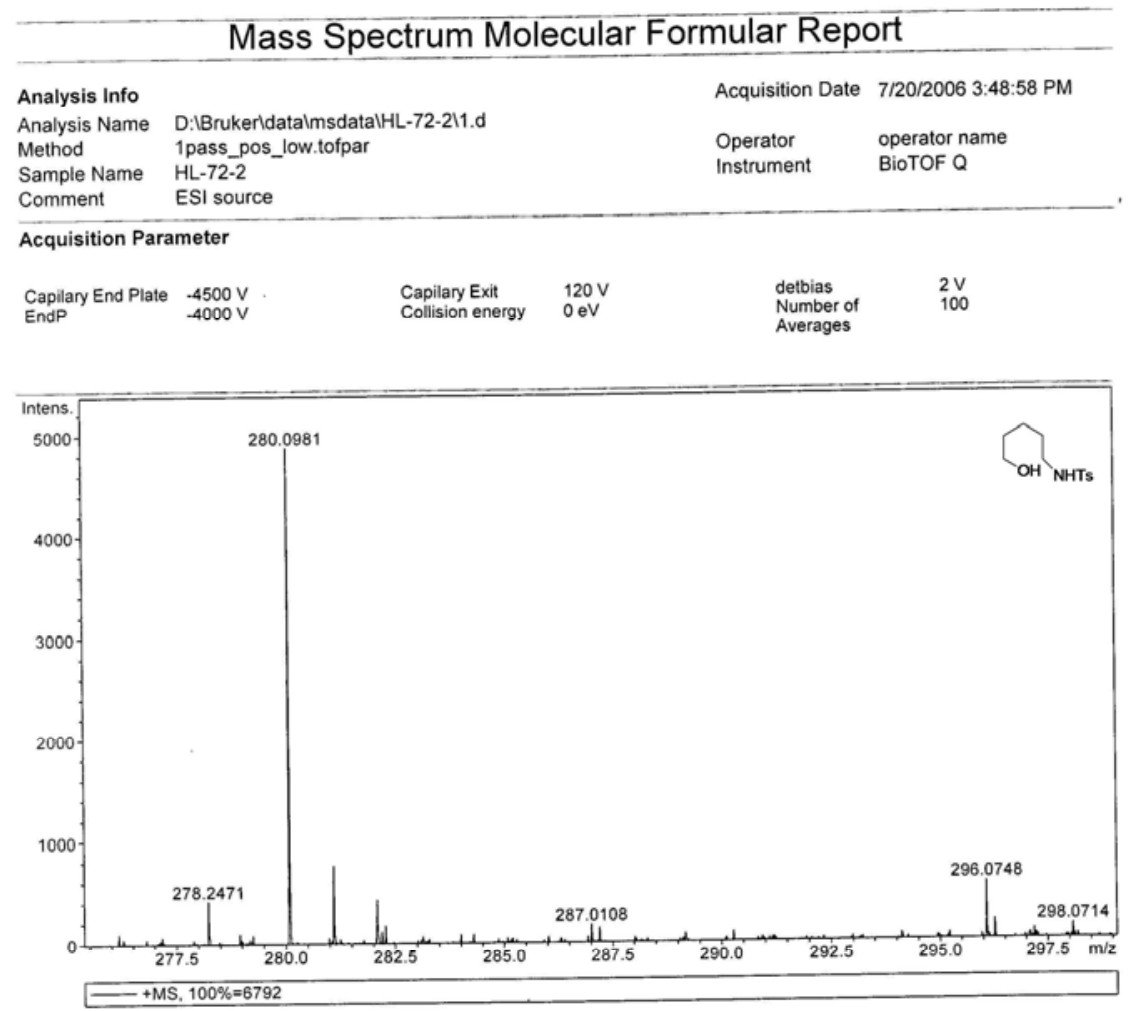

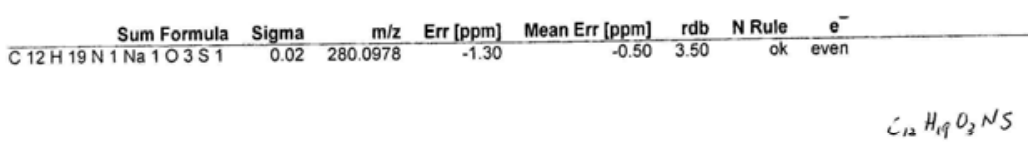




$$
113
$$


HL-75 H1 COC13 2005-6-6

Pulse Sequence: s2pul

$\mathrm{OH} \mathrm{NHTs}$

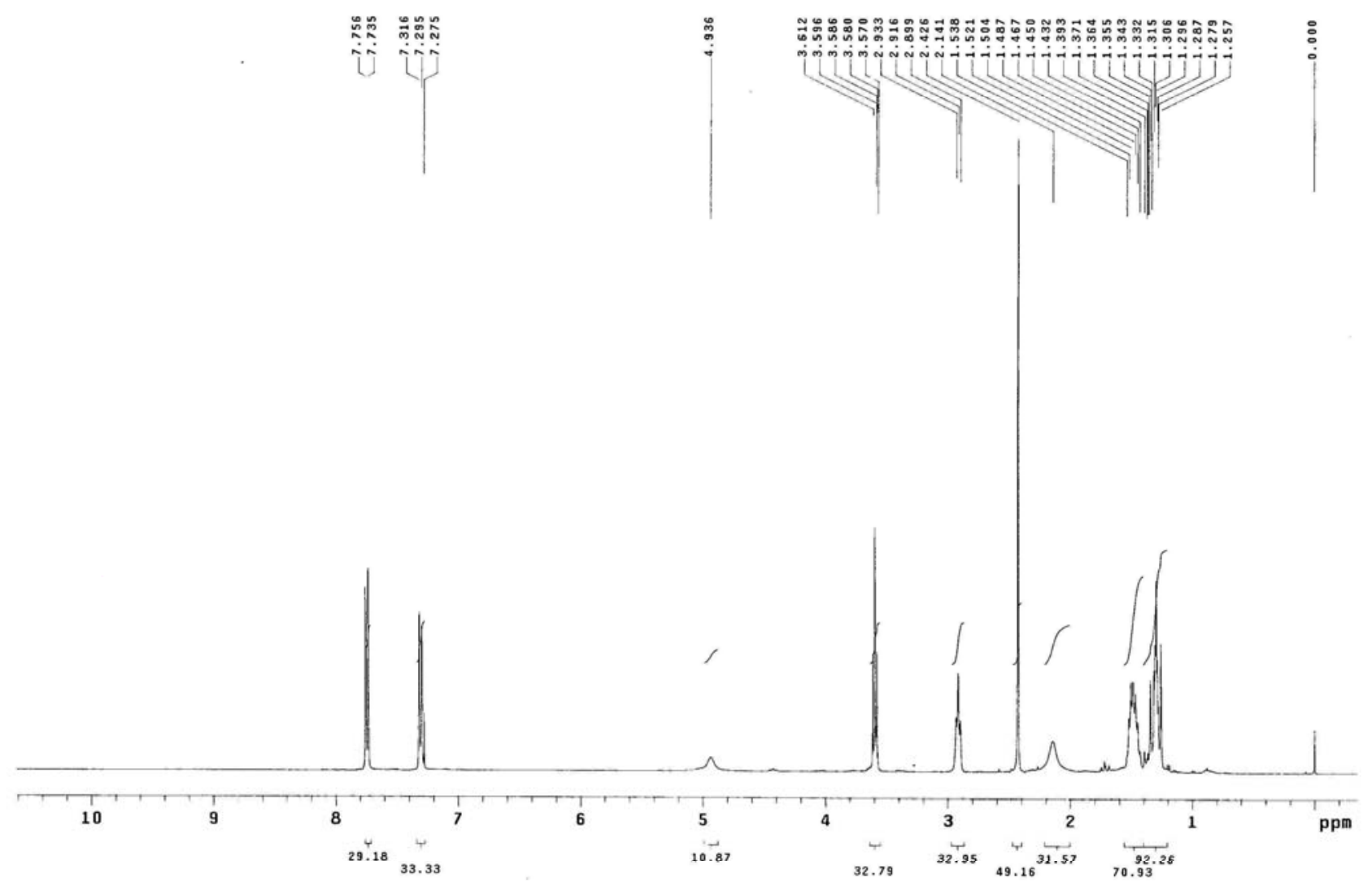




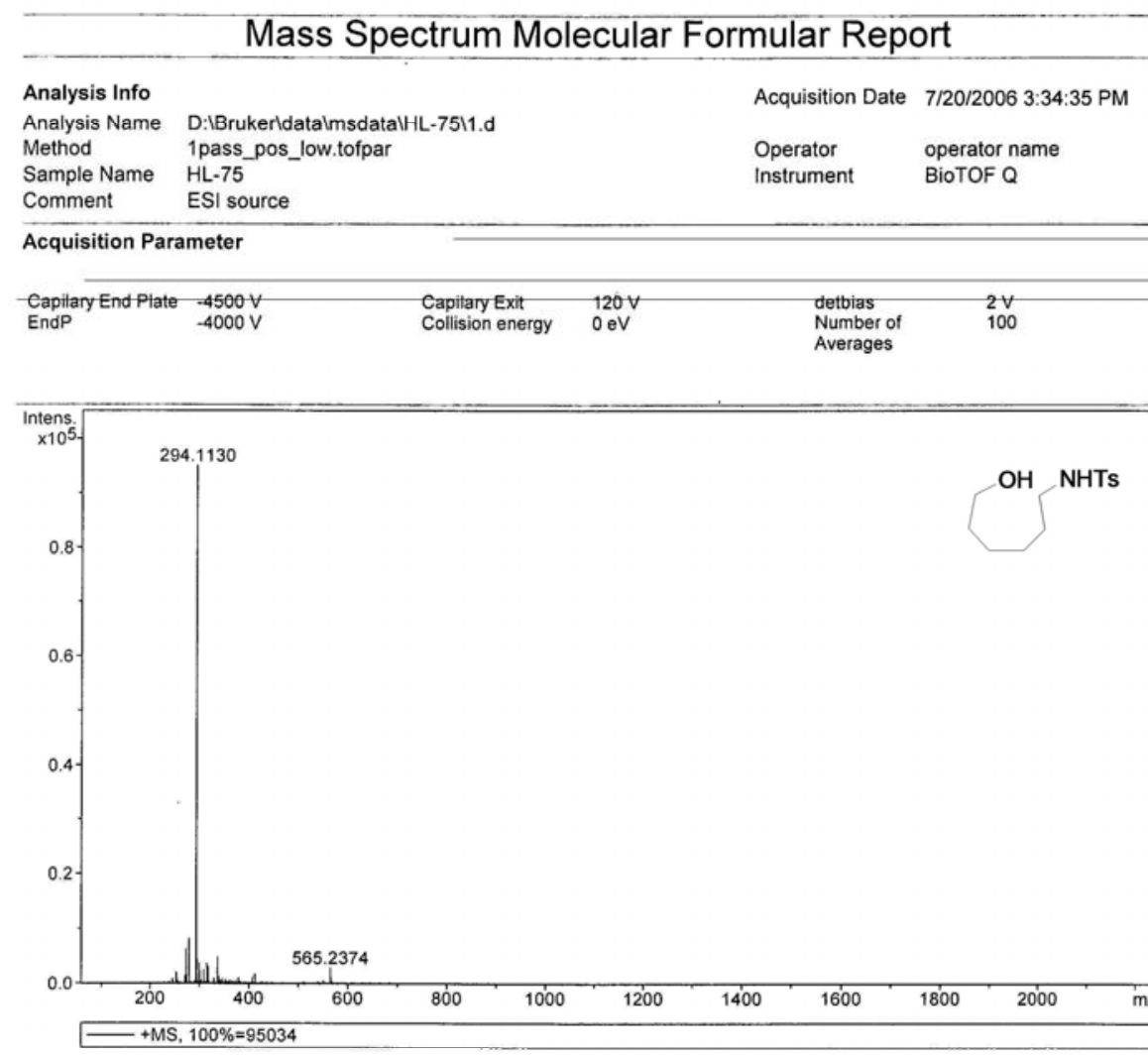

\begin{tabular}{rrrrrrrr} 
Sum Formula & Sigma & $\mathrm{m} / \mathbf{z}$ & Err [ppm] & Mean Err [ppm] & rdb & NRule & $e^{-}$ \\
\hline $\mathrm{C} 13 \mathrm{H} 21 \mathrm{~N} 1 \mathrm{Na} 103 \mathrm{~S} 1$ & 0.01 & 294.1134 & 1.36 & -0.86 & 3.50 & ok & even
\end{tabular} 
HL-76-1-COCL3-H1-2006-7-20

Pulse Seguence: s2pul

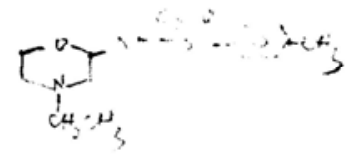

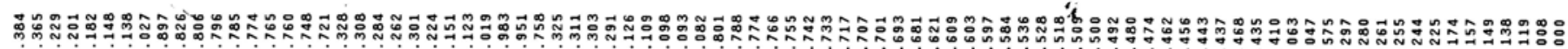

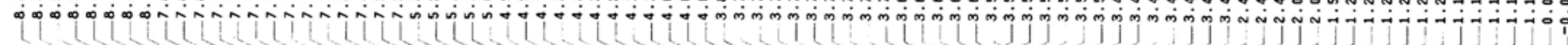

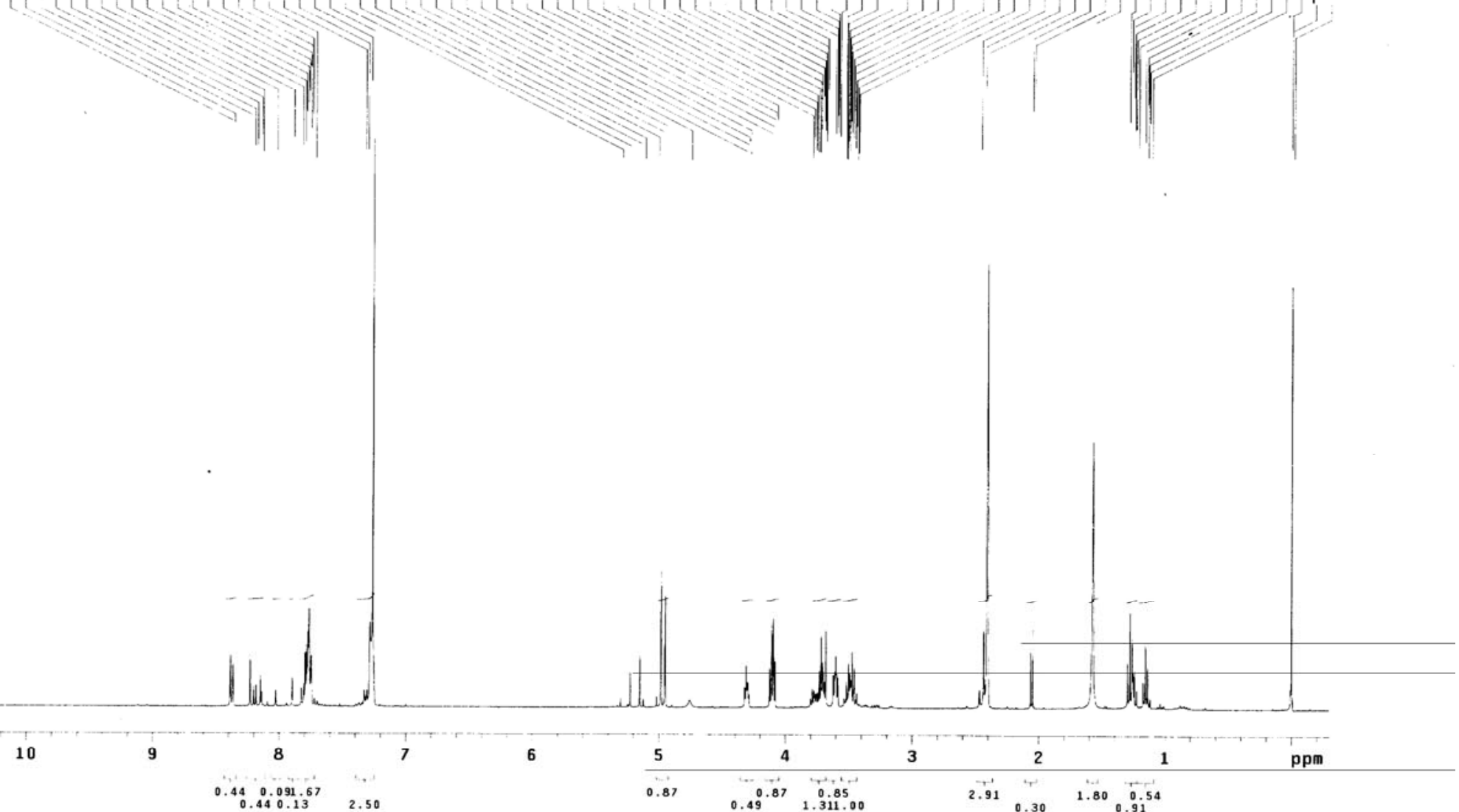




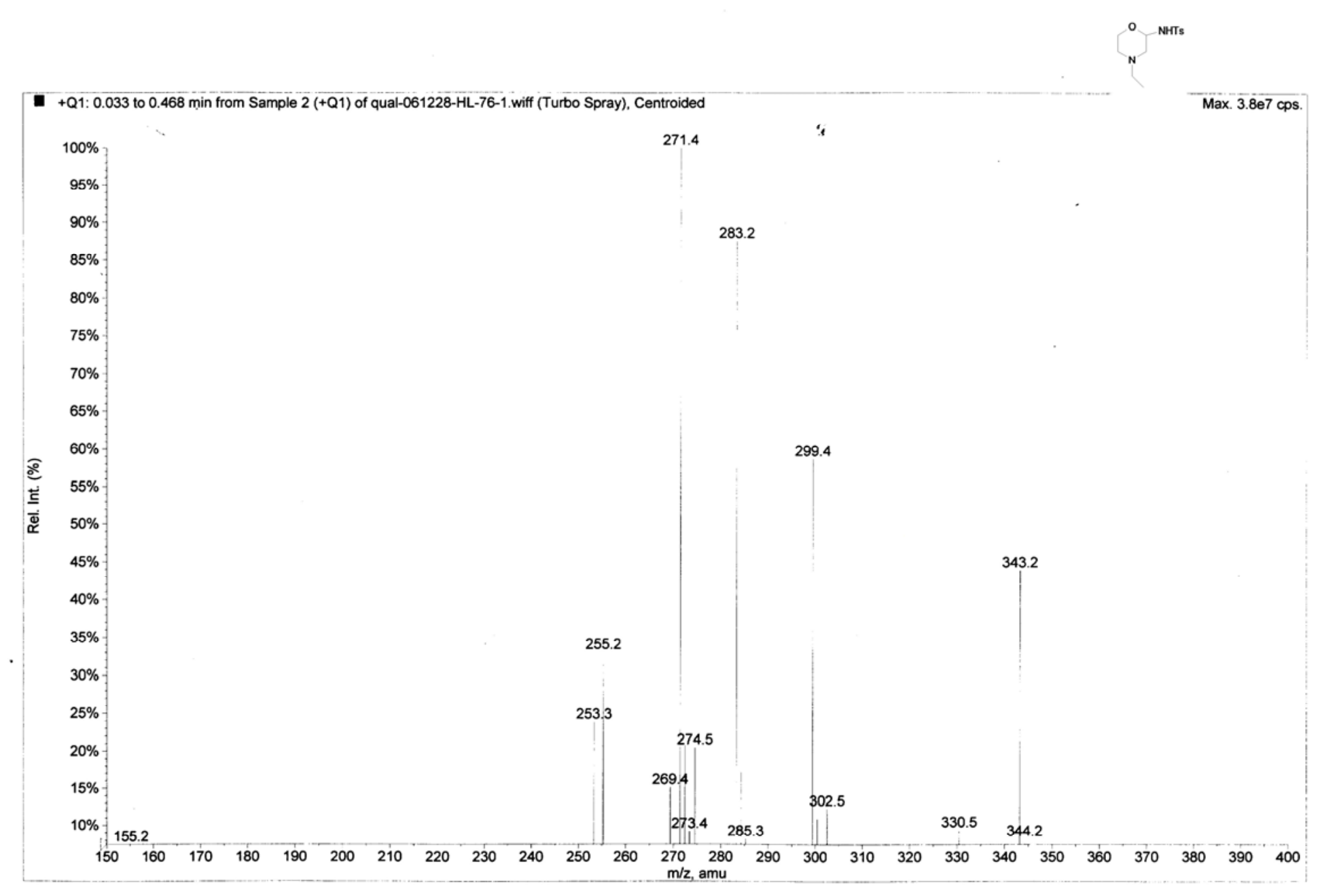




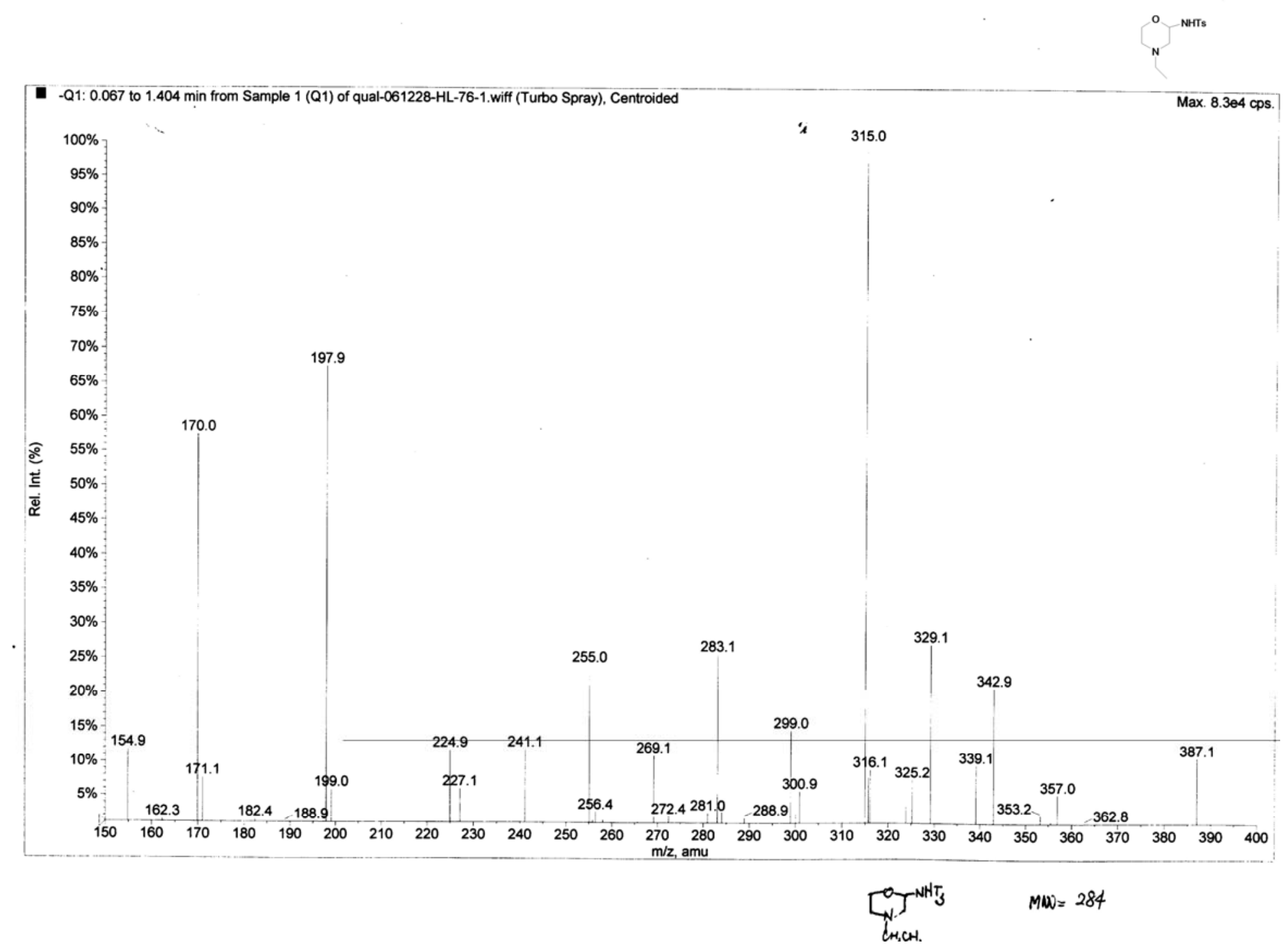



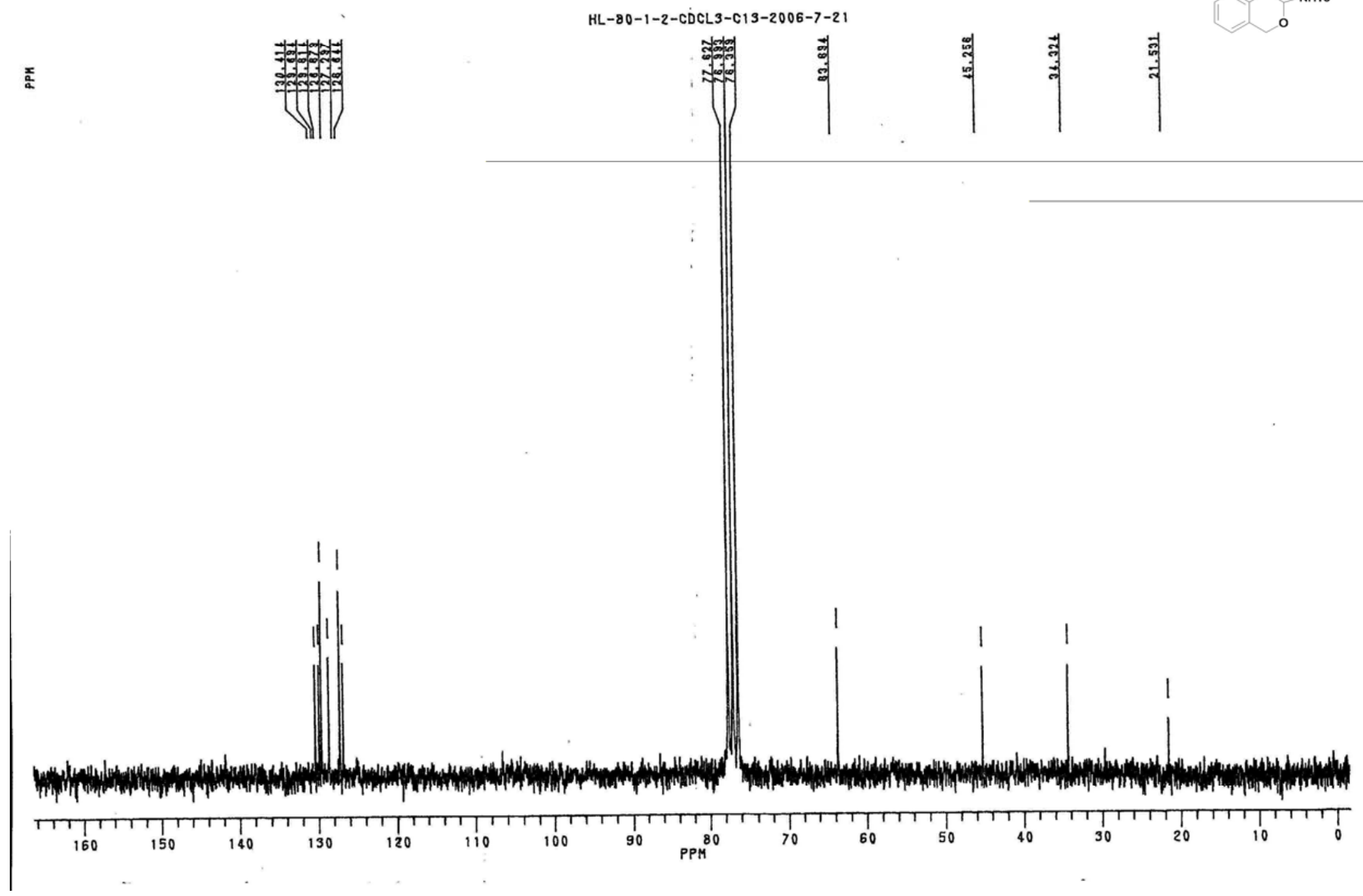

42 


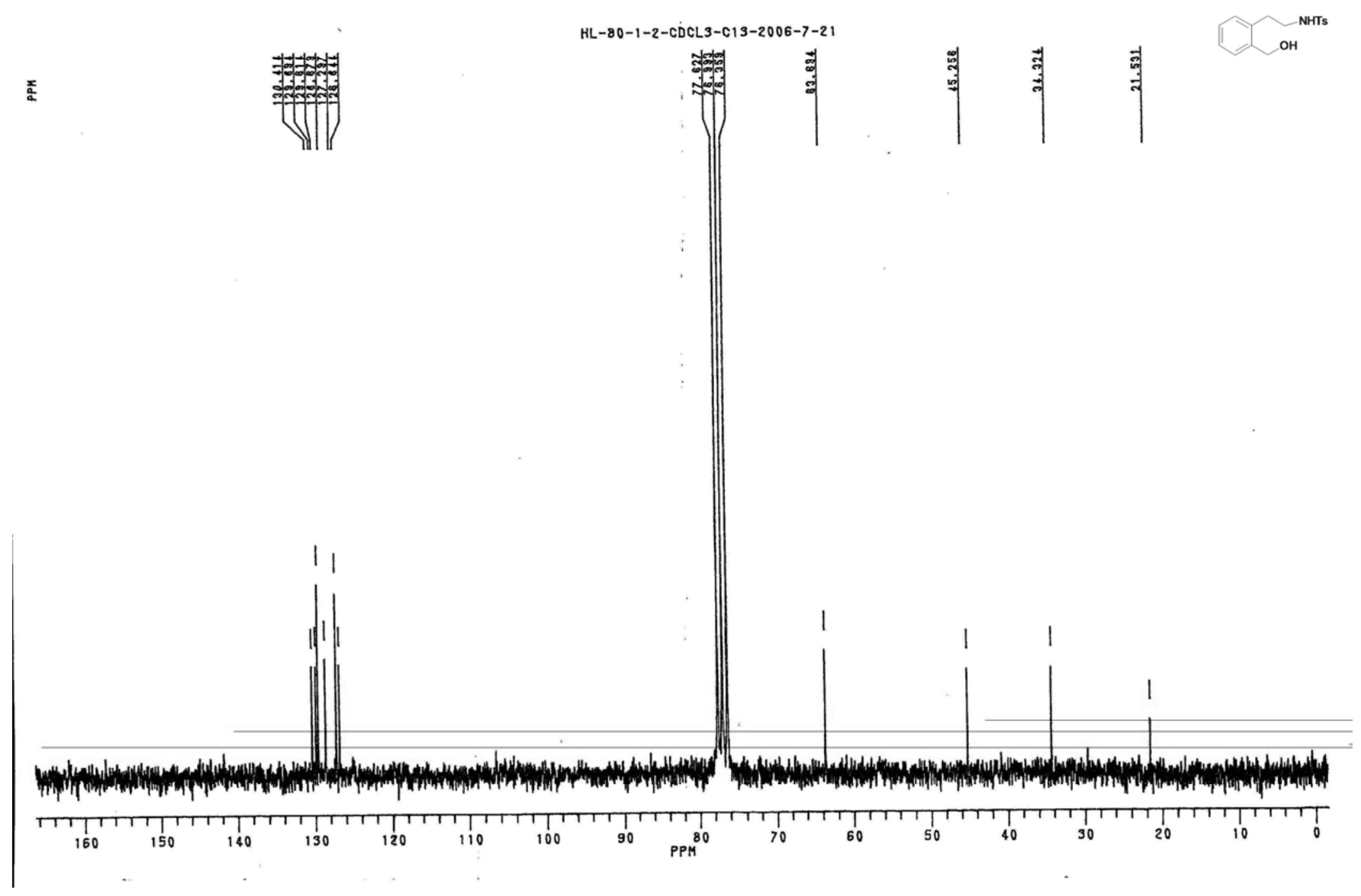


HL-80-1-2-COCL3-H1-2006-7-19

Pulse Sequence: s2pul

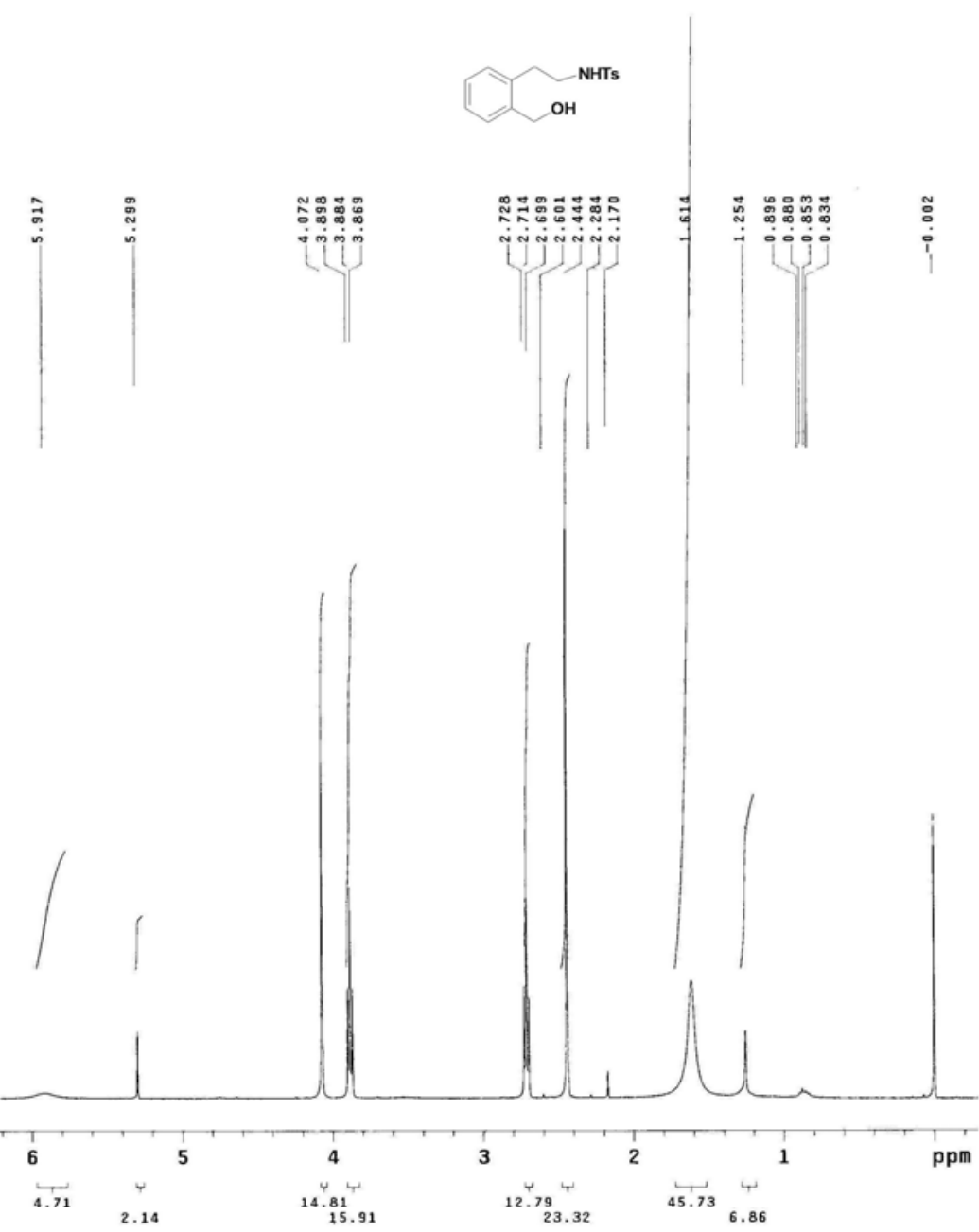




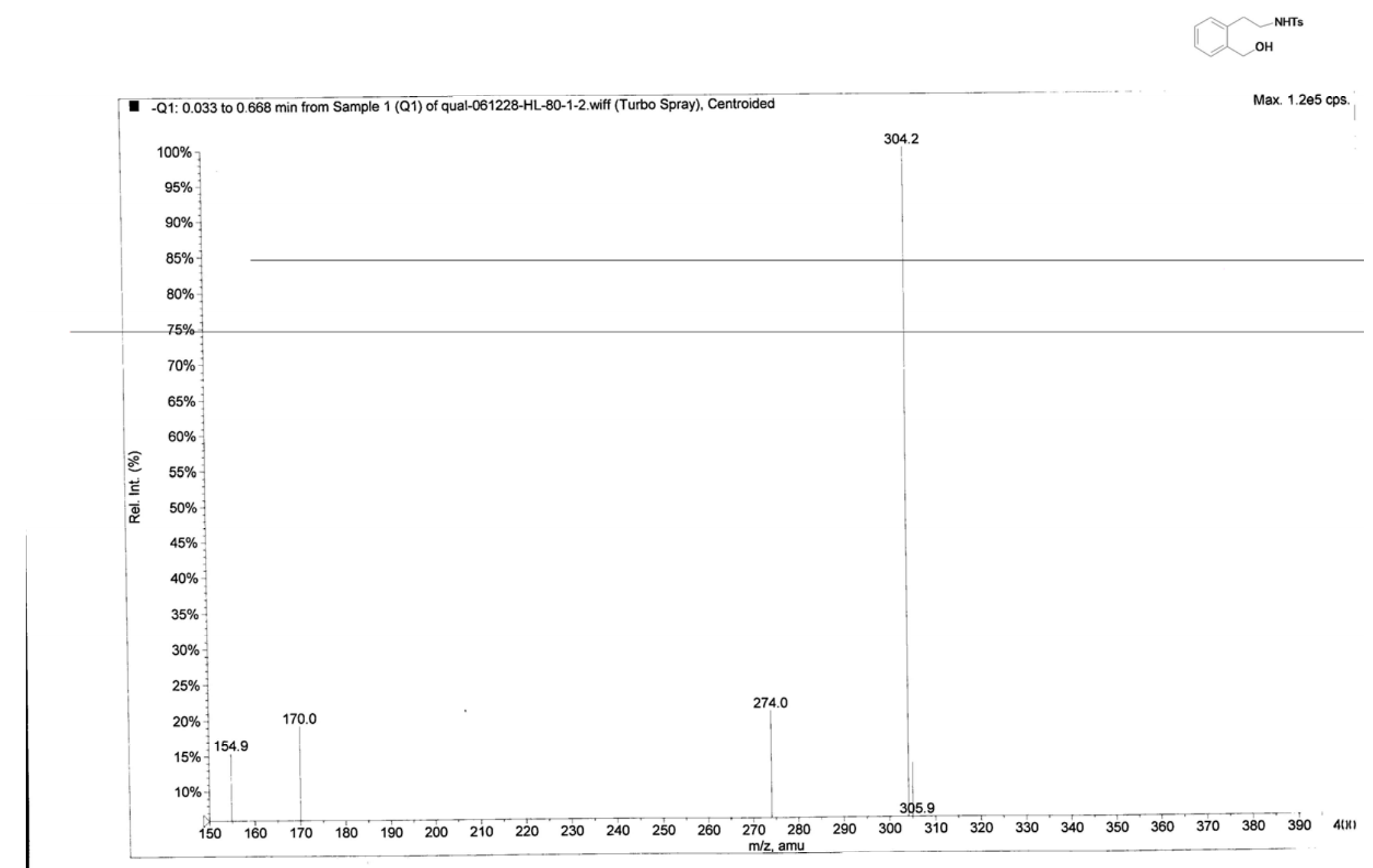


HL-80-1 H1 $\quad \operatorname{COC} 13 \quad 2006-6-20$

Pulse Sequence: s2pul

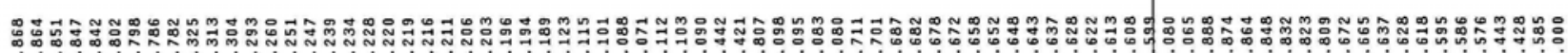

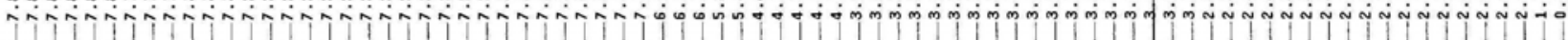

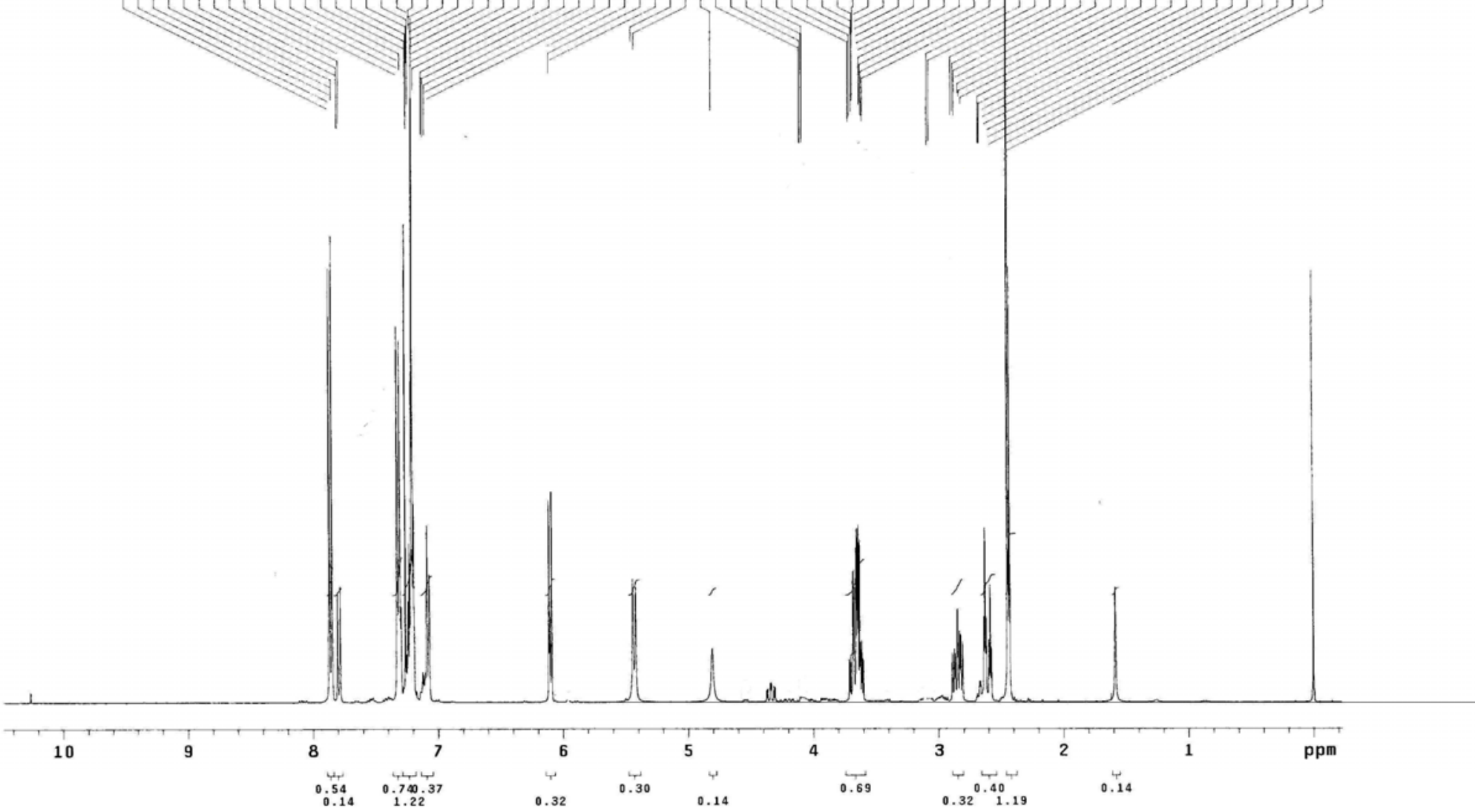




\section{Mass Spectrum Molecular Formular Report}

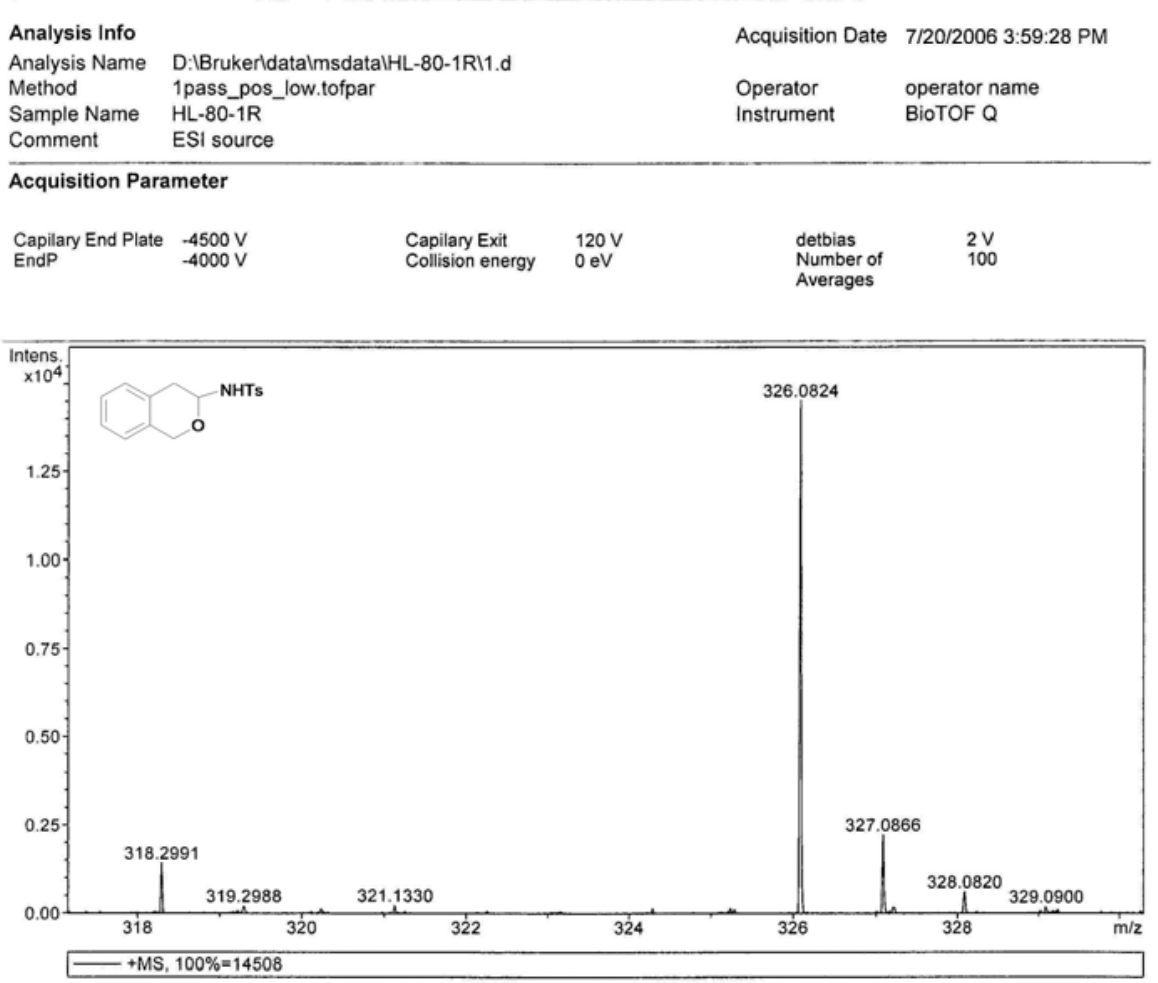

\begin{tabular}{rrrrrrrr} 
Sum Formula & Sigma & $\mathrm{m} / \mathbf{z}$ & Err [ppm] & Mean Err [ppm] & rdb & N Rule & $e^{-}$ \\
\hline C 16H 17 N1 Na 10 3S 1 & 0.02 & 326.0821 & -0.86 & -1.79 & 8.50 & ok & even
\end{tabular} 


$$
\text { II }
$$


HL-80-3-CDCL3-DEPT-2006-7-17
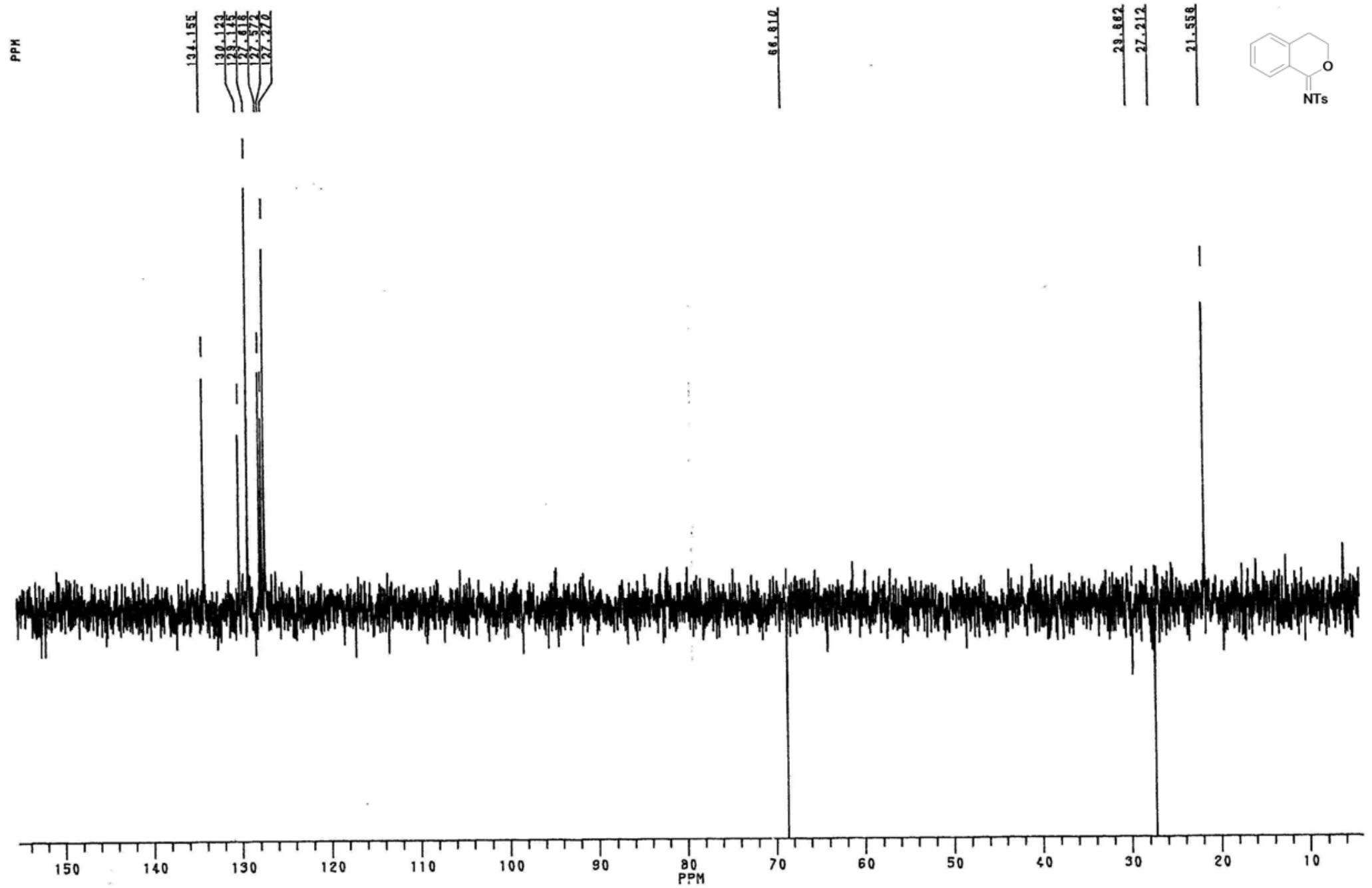


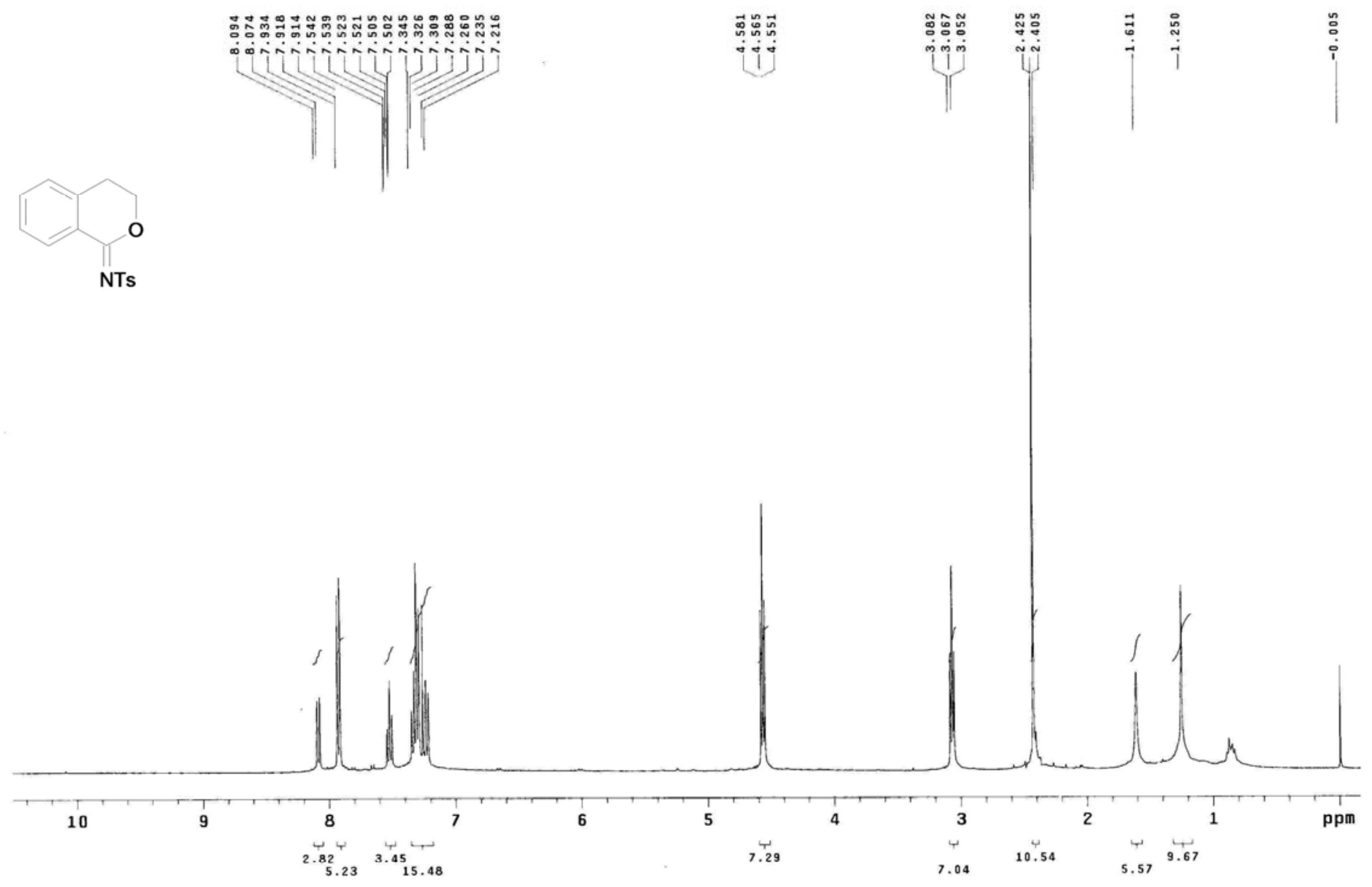


HL-84-3-CDCL3-C13-2006-10-18

Pulse Sequence: s2pul

$\mathrm{TsHN} \bigcirc \mathrm{OH}$
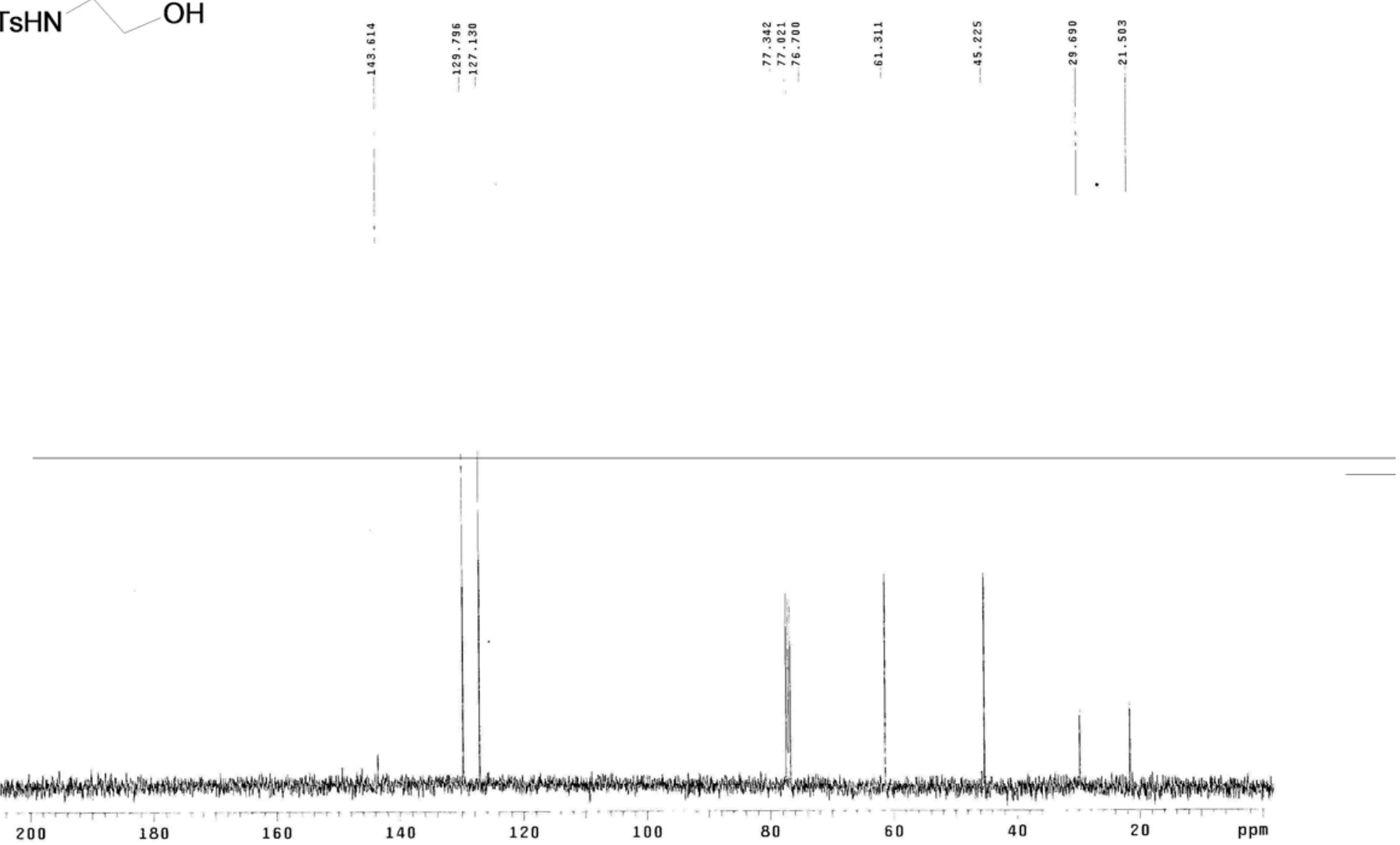
HL-84-3 H1 CDC13 2006-10-11

Pulse Sequence: s2pul

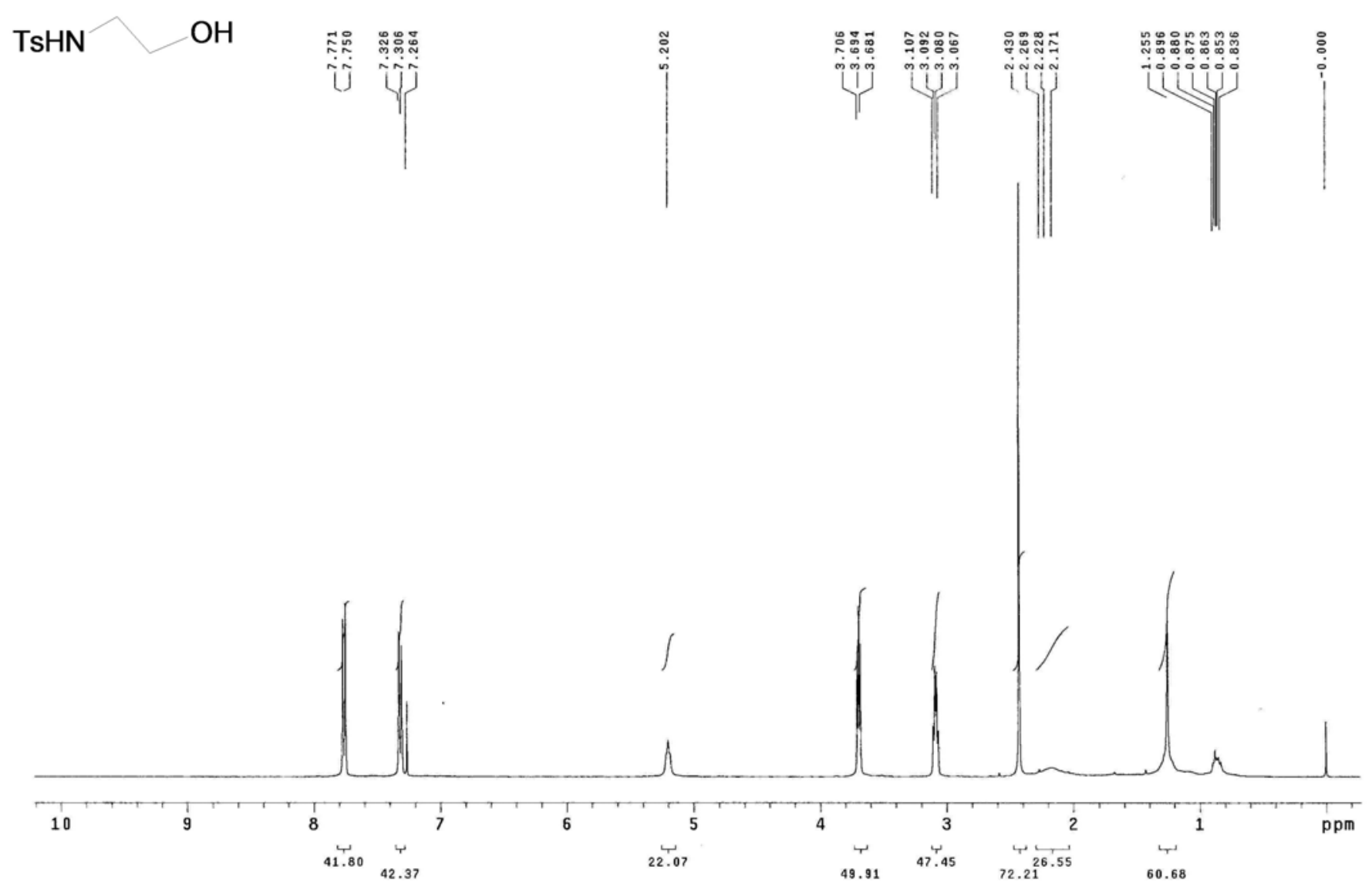

